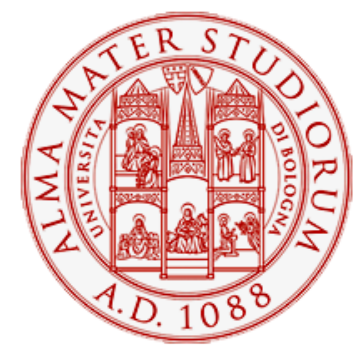

Alma Mater Studiorum - Università di Bologna DEPARTMENT OF ECONOMICS

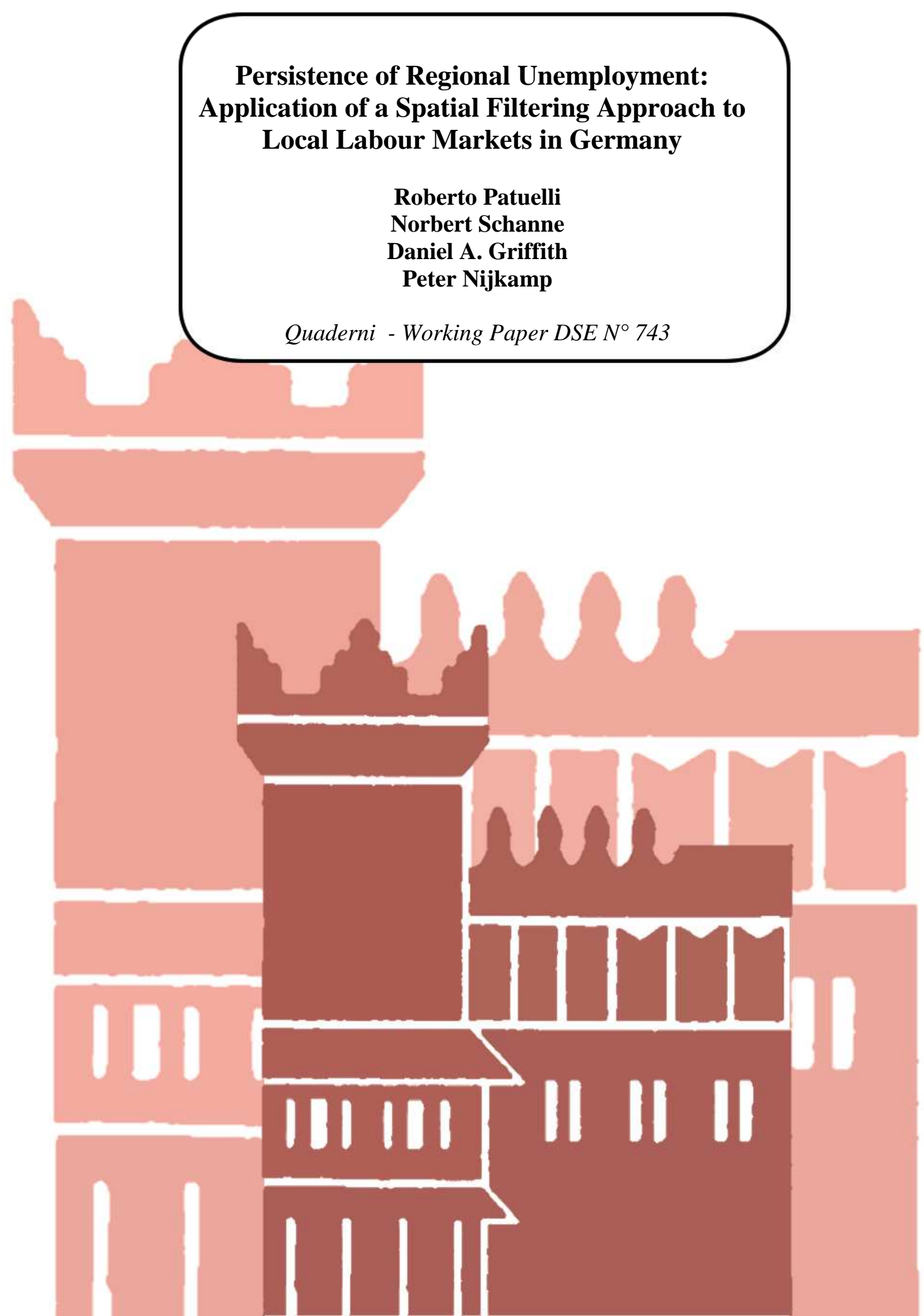




\title{
Persistence of Regional Unemployment: Application of a Spatial Filtering Approach to Local Labour Markets in Germany
}

\author{
Roberto Patuelli, ${ }^{1}$ Norbert Schanne, ${ }^{2}$ \\ Daniel A. Griffith, ${ }^{3}$ and Peter Nijkamp ${ }^{4}$
}

${ }^{1}$ Department of Economics, Faculty of Economics-Rimini, University of Bologna, Italy; Institute for Economic Research (IRE), University of Lugano, Switzerland; The Rimini Centre for Economic Analysis (RCEA), Italy. Email: roberto.patuelli@unibo.it

2 Institute for Employment Research (IAB), Nuremberg, Germany. Email: norbert.schanne@iab.de

3 School of Economic, Political and Policy Sciences, University of Texas at Dallas, USA. Email: dagriffith@utdallas.edu

${ }^{4}$ Department of Spatial Economics, VU University Amsterdam, The Netherlands. Email: pnijkamp@feweb.vu.nl

\begin{abstract}
The geographical distribution and persistence of regional/local unemployment rates in heterogeneous economies (such as Germany) have been, in recent years, the subject of various theoretical and empirical studies. Several researchers have shown an interest in analysing the dynamic adjustment processes of unemployment and the average degree of dependence of the current unemployment rates or gross domestic product from the ones observed in the past. In this paper, we present a new econometric approach to the study of regional unemployment persistence, in order to account for spatial heterogeneity and/or spatial autocorrelation in both the levels and the dynamics of unemployment. First, we propose an econometric procedure suggesting the use of spatial filtering techniques as a substitute for fixed effects in a panel estimation framework. The spatial filter computed here is a proxy for spatially distributed region-specific information (e.g., the endowment of natural resources, or the size of the 'home market') that is usually incorporated in the fixed effects parameters. The advantages of our proposed procedure are that the spatial filter, by incorporating region-specific information that generates spatial autocorrelation, frees up degrees of freedom, simultaneously corrects for time-stable spatial autocorrelation in the residuals, and provides insights about the spatial patterns in regional adjustment processes. We present several experiments in order to investigate the spatial pattern of the heterogeneous autoregressive parameters estimated for unemployment data for German NUTS-3 regions. We find widely heterogeneous but generally high persistence in regional unemployment rates.
\end{abstract}

Keywords: unemployment persistence, dynamic panel, hysteresis, spatial filtering, fixed effects JEL codes: C21, C23, R12 


\section{Introduction}

Regional labour market developments mirror the spatial socio-economic dynamics of the economy. Therefore, timely information on the functioning of these markets is of critical importance for regional policy. In particular, panel-type information on the social economic labour markets may be an important sign post for effective policy, as the spatial-temporal evolution of these markets is critical for understanding the emergency and persistence of spatial disparities among regions. Disparities in economic development and welfare within countries (at the regional level) are often bigger than between countries (Elhorst 1995, Taylor and Bradley 1997, Ertur and Le Gallo 2003, Patuelli 2007; see, for example, the cases of Germany and Italy), and they often show typical geographical/spatial structures. Consequently, spatial disparities have for decades been a source of policy concern and applied research (for a recent overview of this field, see Kochendörfer-Lucius and Pleskovic 2009). Spatial disparities occur in both developed and developing countries; their genesis may date back far in history, while their removal may take generations.

For example, Germany faced, in the first semi-decade after reunification, an increase in unemployment, from 2.6 million people in 1991 to 4.3 million people in 1997 - or, including the hidden reserve, from 3 millions to 5.6 millions (Fuchs, et al. 2010). Unemployment remained, with only slight movements, at the same level for roughly 10 years, until the rapid decline after the 2005 reforms. In the period from 2006 to 2010 unemployment dropped again to the level of the early 1990s, despite the credit crunch. Throughout the high-unemployment period from 1995 to 2005, the unemployment rate in East Germany was 9 to 11 percentage points higher than the unemployment rate in West Germany; however, as we show later in the paper, there were large disparities within West German unemployment rates as well. In particular, in the two most recent years, the East-West disparities in the unemployment rates have diminished.

Underperforming regions imply, for a (redistributive) state, the need to allocate a higher share of public spending to those regions, eventually creating distortions in the redistribution of tax revenues and increasing conflicts with local policy makers and the public. Additionally, high unemployment has historically been linked to a number of socioeconomic problems, such as single-parent households, underperformance of students in school, truancy rates, and more (Armstrong and Taylor 2000). Persistently high unemployment rates have been shown to be correlated with high shares of long-term unemployment and outmigration (for example, recent data for Southern Italy show an increase in the outmigration - toward the North - of the top university graduates; see SVIMEZ 2009). 
With regard to regional unemployment disparities, policy makers need, in order to correctly target their actions and policies, to understand two aspects of such disparities: (a) the determinants of 'equilibrium' unemployment and its variation; and, (b) the region-specific and the cross-regional dynamics of unemployment. The determinants of unemployment have been studied extensively in the regional economic literature (Taylor and Bradley 1997, Badinger and Url 2002, Aragon, et al. 2003, Elhorst 2003, Niebuhr 2003, Basile and De Benedictis 2008, Nijkamp 2009, Zenou 2009, Moretti 2010, Oud, et al. 2010). Some attention has been as well devoted to the internal dynamics of regional unemployment, and to each region's sensitivity to shocks, seasonal factors, and persistence of unemployment. The available literature is mostly focusing on a macroeconomic setting, such as in a "nonaccelerating inflation rate of unemployment (NAIRU)' or in a (conditional/unconditional) 'convergence towards a natural rate of unemployment' perspective (following the approach of Blanchard and Summers 1986; see, for example, Decressin and Fatás 1995, Song and Wu 1997, Bayer and Juessen 2007, Garcia-del-Barrio and Gil-Alana 2009, Tyrowicz and Wójcik $2010 b$, c, a). From a technical perspective, these studies generally test for unit roots in the unemployment series. ${ }^{1}$ However, they suffer from the major drawbacks of treating regions as homogeneous and/or cross-sectionally independent: they consider neither spatial correlation of shocks nor spatially structured heterogeneity in the adjustment process.

Similarly, the correlation of unemployment rates in space - that is, between neighbouring regions - has been studied both in an exploratory/descriptive fashion (Molho 1995, LópezBazo, del Barrio and Artis 2002, Cracolici, Cuffaro and Nijkamp 2007, Mayor and López 2008, Patuelli, et al. 2010a), and with regard to the determinants of unemployment (Elhorst 1995, Mitchell and Bill 2004, Kosfeld and Dreger 2006, Patacchini and Zenou 2007, Aldashev 2009), using spatial-econometric techniques. However, little effort has been made, aside from in a time series/forecasting context (Schanne, Wapler and Weyh 2009), to decompose the spatial dynamics of unemployment, so that region-specific autoregressive processes (responses to shocks), or region-specific seasonal characteristics can be traced. However, besides the old and general story that regions are not isolated islands, some specific arguments - such as commuting and internal migration, the spatial diffusion of information on vacancies, the (limited) search radius of unemployed persons, which affect the duration (and persistence) of individual unemployment - exist for spatially structured regional

\footnotetext{
Stationarity implies that a series has a distribution with finite variance and that it converges towards its longrun expectation. Convergence between the regions arises only if the regional series have the same long-run expectation. In contrast, non-stationary regional series imply that shocks persist and that in the long-run the cross-regional distribution depends completely on accumulated (random) events.
} 
interdependence in the development of aggregate unemployment. In other words, regions are expected to differ in their degree of persistence, and this heterogeneity is likely to show a spatial pattern.

Policy makers who understand the specific characteristics of a region and of interregional dependencies are able to tackle problems more effectively and to anticipate more accurately necessary responses to aggregate and local shocks. Likewise, a group of (contiguous) regions that share common characteristics has the opportunity to develop common strategies (for example, within a single macro-region, such as a German Bundesland). We stress the need to investigate (break down) the components of region-specific dynamics, from an autoregressive/reaction-to-shocks viewpoint, so as to identify spatial patterns of common characteristics. A similar view was recently expressed by Partridge and Rickman (2010) in their review and discussion of (desirable) developments in CGE modelling.

The empirical research in our study will address the development of regional labour markets over a longer period in Germany. This country offers a unique natural experiment for our purposes, as - in addition to the regular spatial dynamics of an advanced industrial economy the post-reunification effects appear to play a prominent role in the initial distribution of unemployment and the subsequent evolution of spatial disparities in the country, generating a certain amount of regional dynamics. Nevertheless, since unit-root tests are sensitive to structural breaks, it is important to deal properly with the direct impact of reunification. This paper aims to develop a number of autoregressive models for analysing regional unemployment between 1996 and 2004, that is, the period after the direct effect of reunification has fully realized, and before the major labour market reforms, in the 439 German NUTS-3 regions (kreise). These administrative regions can be considered an ideal unit of analysis, because they directly relate to local policy-making choices, for example in public welfare, ${ }^{2}$ in terms of attracting capital- or labour-intensive industries through the provision of a productive environment, infrastructure, enterprise zones, or by subsidizing desired economic activities. ${ }^{3}$ We estimate autoregressive effects specific to both each administrative region and different urbanization and agglomeration degrees of regions. In addition to a standard fixed effects (FE)/individual slopes estimation, we propose an econometric procedure suggesting the use of spatial filtering (SF) techniques as a substitute

\footnotetext{
${ }^{2}$ Until 2004, two parallel benefit systems for long-term unemployed coexisted. The 'Arbeitslosenhilfe' was administered by the local departments of the Federal Employment Agency, while the 'Sozialhilfe' was under the responsibility of the NUTS-3 authorities (kreise).

3 Although the major part of subsidies is distributed by the federal states, the national government or the European Union, many programmes require co-funding from the local authorities, and availability depends on criteria often calculated at the NUTS-3 level.
} 
for region-specific parameters in a panel estimation framework. The spatial filter is a proxy for spatially distributed region-specific information (e.g., the endowment of natural resources or the size of the 'home market') that is usually incorporated in the FE or in region-specific slope parameters. The approach presented here is beneficial, because it allows considerable savings in terms of degrees of freedom. Most importantly, the spatial filter provides a straightforward interpretation - as the linear combination of orthogonal spatial patterns - of the FE components surrogate. By incorporating region-specific information that generates spatial autocorrelation and dynamics, our procedure provides new insights about the spatial patterns that make it interesting to adopt the approach also for the analysis of other spatiotemporal processes, such as GDP growth/convergence, house price diffusion, and spread of diseases.

In this paper, we present several experiments investigating the spatial patterns of autoregressive parameters estimated for the unemployment rates of German NUTS-3 regions. Our findings show that - on average - unemployment rates are rather persistent and that the levels of persistence have an identifiable spatial structure. The proposed methodological approach also shows to be a promising tool for the analysis of regional dynamics. Additionally, we propose a model based on spatial regimes, which allows to decompose the dynamic processes of regional unemployment rates according to agglomeration/urbanization criteria, rather than to the well-known - but oversimplifying - East-West Germany division.

The remaining part of the paper is structured as follows. Section 2 describes the analytical design of the model used in our study. Sections 3 and 4 present the dataset used and the results obtained, respectively. Finally, Section 5 provides a rejoinder and conclusive remarks.

\section{Analytical Design of the Model}

\subsection{The Traditional Approach}

The current standard approach to analyse the persistence of unemployment or, in a multiregion context, its convergence speed (see, for a recent overview, Lee and Chang 2008) is to estimate a system of $\mathrm{AR}(1)$ processes, and to test each single equation as well as the entire system of equations for unit roots. Here, the basic equation for unemployment $u$ in region $i$ is given by Equation (1): 


$$
u_{i, t}=\alpha_{i} u_{i, t-1}+\mu_{i}+s_{i, t}+\varepsilon_{i, t},
$$

where $\mu_{i}$ denotes the average unemployment, ${ }^{4} s_{i, t}$ its seasonal component, and $\varepsilon_{i, t}$ an i.i.d. mean-zero random disturbance. Stacked over all regions, this set can be written as the following system of equations:

$$
\mathbf{U}_{t} \mathbf{l}_{n}=\mathbf{U}_{t-1} \mathbf{A}_{n}+\mathbf{M}_{n}+\mathbf{S}_{t}+\varepsilon_{t}
$$

where $\mathbf{U}_{t}=\operatorname{diag}_{i=1}^{n}\left(u_{i, t}\right)$, is the $n \times n$ diagonal matrix of unemployment rates at time $t, \mathbf{A}_{n}=$ $\left(\alpha_{1}, \ldots, \alpha_{N}\right)^{\prime}$ and $\mathbf{M}_{n}=\left(\mu_{1}, \ldots, \mu_{N}\right)^{\prime}$ are $n \times 1$ column vectors of parameters, $\mathbf{S}_{t}=\left(S_{1, t}, \ldots, S_{N, t}\right)^{\prime}$ is an $n \times 1$ column vector (generated from the $n \times 3$ matrix of parameters corresponding to the seasonal dummies, multiplied by the $3 \times 1$ matrix containing the seasonal dummies), $\mathbf{1}_{n}=$ $(1, \ldots, 1)^{\prime}$ is a unit vector of length $n$, and $\varepsilon_{t}=\left(\varepsilon_{1, t}, \ldots, \varepsilon_{N, t}\right)^{\prime}$ is the $n \times 1$ vector of residuals. The subscript $n$ in $\mathbf{A}_{n}$ and $\mathbf{M}_{n}$ denotes the length of the parameter vectors. Vectors and matrices with subscript $t$ always have length $n . \mathbf{M}_{n}$ is equivalent to $\mathrm{FE}$ in a panel framework. ${ }^{5}$

If the autoregressive parameter $\alpha_{i}$ is smaller than 1 in absolute value, the impact of a "shock" $\varepsilon_{i, t}$ will vanish over time, and the series will converge to its long-run expectiation. In contrast, if $\alpha_{i}$ equals one, the process in region $i$ has a unit root. A single equation is tested for stationarity by augmented Dickey-Fuller (ADF) tests, or by Phillips-Perron (PP) tests; likewise, various tests derived for panels or systems that rely as well on subtracting lagged unemployment from both sides of Equation (2) require the following form of Equation (2):

$$
\left(\mathbf{U}_{t}-\mathbf{U}_{t-1}\right) \mathbf{l}_{n}=\mathbf{U}_{t-1}\left(\mathbf{A}_{n}-\mathbf{l}_{n}\right)+\mathbf{M}_{n}+\mathbf{S}_{t}+\varepsilon_{t},
$$

Next, we may test if the elements of $\left(\mathbf{A}_{n}-\mathfrak{l}_{n}\right)$ are, individually or jointly, significantly less than zero. ${ }^{6}$ Some procedures test the entire set of parameters directly (for example, Sarno and Taylor 1998), whereas others combine the individual $t$-statistics to form a joint test statistic (see Maddala and Wu 1999 or Im, Pesaran and Shin 2003). As an alternative, restrictions may

\footnotetext{
${ }^{4}$ We assume that unemployment does not have a deterministic trend.

5 For small time dimensions, the estimates of the autoregressive parameters are typically downward biased. With individual parameters, the Hurwicz bias is $\tilde{\alpha}_{i}-\alpha_{i}=-\left(1+3 \alpha_{i}\right) / T$. The Nickell bias, $\tilde{\alpha}-\alpha=-(1+\alpha) /(T-1)$, for a common parameter across the regions $\alpha_{1}=\ldots=\alpha_{n}=\alpha$ has a smaller size than the Hurwicz bias (Nickell 1981). However, it can be seen that both converge towards zero when $T$ goes to infinity. ${ }^{6}$ The parameters $\alpha_{i}-1$ follow, under the null hypothesis of a non-stationary process, a non-normal degenerate distribution, typically a Wiener process (also denoted as Brownian motion).
} 
be imposed on the parameter, enabling a test only for stationarity of the average autoregressive process, as in Levin et al. (2002), or for the stationarity of a limited number of regime-specific processes (also referred to as the 'convergence clubs' hypothesis).

Regarding the validity of panel unit-root tests, most of these procedures require the time dimension to be sufficiently large in order to converge and not to be plagued by the so-called Nickell bias arising in panels with a small time dimension (Nickell 1981) or by the Hurvicz bias in short times series. Moreover, Equations (2) and (3) are only estimable in a seemingly unrelated regression (SURE) form (that is, in a specification that allows for simultaneously correlated errors) when the number of regions is small. Else one has to assume independence of the regions, resulting in equation-wise unit-root tests with low efficiency/power. Nonetheless, cross-sectional correlation seems rather plausible, in particular when considering small spatial units, and therefore taking this structure into account in the error term $\varepsilon_{t}$ is preferable.

Cross-sectional (spatial) correlation arises not only in contemporaneous shocks, but also in levels and trends (as shown in TABLE 1), in seasonal patterns, or in the adjustment speed. On the one hand, these spatial patterns or correlations could likewise be utilized to get better more efficient, more powerful, less demanding in terms of degrees of freedom, and large- $N$, small- $T$ consistent - estimates of the average convergence speed. On the other hand, knowledge about spatial interdependence between the structures of a time-series average/trend, seasonality, and autoregressive properties - may be of direct interest as well.

TABLE 1: Descriptive statistics of regional unemployment, 1996-2004

\begin{tabular}{lcccccc}
\hline Region & Mean & St. dev. & 1st quartile & Median & 3rd quartile & MI \\
\hline \multicolumn{7}{c}{ Unemployment rates (levels, in \%) } \\
\hline Germany & 11.8 & 5.5 & 7.6 & 10.1 & 15.4 & 0.903 \\
East & 19.4 & 3.5 & 17.0 & 19.3 & 21.8 & \\
North & 11.1 & 2.8 & 9.0 & 10.7 & 13.0 & \\
South & 8.1 & 2.5 & 6.2 & 7.7 & 9.5 & 0.623 \\
& & & First differences (in \%) & \\
\hline Germany & 0.01 & 1.21 & -0.43 & 0.11 & 0.59 & 1.22 \\
East & 0.06 & 1.76 & -0.88 & 0.30 & 0.40 & \\
North & -0.01 & 0.89 & -0.34 & 0.06 & 0.60 & \\
South & -0.06 & 0.88 & -0.72 & -0.07 & \\
\hline
\end{tabular}


In the following subsection, we propose an alternative approach to estimating Equation (2), which decomposes the autoregressive processes according to exogenous spatial patterns that are representative of accessibility/contiguity relations between the regions studied. The benefit is twofold: (a) we obtain an explicit model of the spatial patterns in unemployment without being over-restrictive by imposing (probably erroneous) regime-specific constraints; and, (b) we are able to estimate more parsimoniously while covering the most relevant spatial structures. $^{7}$

\subsection{Spatial Filtering}

A wide array of methods, as well as several dedicated 'spatial' econometric procedures, for the statistical analysis of georeferenced data is available in the literature. Most commonly employed, spatial autoregressive techniques (see, for example, Anselin 1988) model interregional dependence explicitly by means of spatial weights matrices that provide measures of the spatial linkages between values of georeferenced variables, with a structure similar to serial correlation in time-series econometrics.

An alternative approach to spatial autoregression, modelling spatial autocorrelation in the mean response rather than in the variance, is the use of spatial filtering (SF) techniques (Getis and Griffith 2002). Their advantage is that the studied variables (which are initially spatially correlated) are split into spatial and non-spatial components. Then these components can be employed in a linear regression framework. This conversion procedure requires the computation of a 'spatial filter'.

The SF technique introduced by Griffith (2003) is based on the computational formula of Moran's I (MI) statistic. ${ }^{8}$ This eigenvector decomposition technique extracts $n$ orthogonal, as well as uncorrelated, numerical components from the $n \times n$ modified spatial weights matrix:

7 This claim clearly needs to be further explored by simulation evidence showing that SF is a suitable substitute/approximation of the fixed effects. Preliminary simulation results by the authors suggest that the SF and SFGWR are fully competitive - unless $N$ or $T$ tend to infinite - with mainstream econometrics methods such as bias-corrected LSDV (Bun and Carree 2005) and Blundell and Bond (1998), in terms of parameter estimate bias.

8 Moran's I is calculated as follows:

$I=\frac{N \sum_{i} \sum_{j} w_{i j}\left(x_{i}-\bar{x}\right)\left(x_{j}-\bar{x}\right)}{\left(\sum_{i} \sum_{j} w_{i j}\right) \sum_{i}\left(x_{i}-\bar{x}\right)^{2}}$,

where, in the case of a set of $n$ regions, $x_{i}$ is the value of the generic variable $x$ in region $i$, and $w_{i j}$ is the cell $(i$, $j$ ) of a spatial weights matrix $\mathbf{W}$, indicating the proximity of each pair of regions $i$ and $j$. 


$$
\mathbf{W}=\left(\mathbf{I}_{n}-\mathfrak{u}^{\prime} / n\right) \mathbf{C}\left(\mathbf{I}_{n}-\mathfrak{u}^{\prime} / n\right)
$$

where $\mathbf{I}_{n}$ is an identity matrix of dimension $n, \imath$ is an $n \times 1$ unit vector, and $\mathbf{C}$ is a spatial weights matrix ${ }^{9}$ representing the spatial relation between each pair of regions; here we use a binary first-order contiguity (C-coding rook) matrix where element $c_{i j}$ equals 1 if regions $i$ and $j$ have a common border, and 0 otherwise. Matrix $\left(\mathbf{I}_{n}-\mathfrak{u}^{\prime} / n\right)$ is the standard projection matrix found in the multivariate statistics and regression literature. Because matrix $\mathbf{C}$ is preand post-multiplied by the projection matrix [see Equation (4)], these eigenvectors are centred at zero. The eigenvectors extracted are in a decreasing order of spatial autocorrelation, and the first corresponds to the largest eigenvalue of $\mathbf{W}$. Thus, the first two eigenvectors computed $\left(\mathbf{E}_{1}\right.$ and $\mathbf{E}_{2}$ ) often identify map patterns along the cardinal points (that is, some rotated version of the major North-South and East-West patterns). Eigenvectors with intermediate values of MI display regional map patterns, whereas eigenvectors with smaller values of MI display local map patterns. The set of relevant eigenvectors - those explaining the spatial pattern in the variable of interest - can be found by regressing the dependent variable on the eigenvectors in a stepwise fashion, retaining the significant eigenvectors (or eliminating the insignificant ones). The linear combination of selected eigenvectors and their corresponding parameter estimates define the spatial filter for the variable of interest. In an autoregressive setting (where no covariates are employed), residuals obtained with stepwise regression constitute the spatially filtered component of the georeferenced variable examined (see Griffith 2000). The eigenvectors can be seen as independent map patterns that coincide with the latent spatial autocorrelation of a given georeferenced variable, according to a given spatial weights matrix. Moreover, they can work as proxies for omitted variables that show a certain coincidence or similarity regarding their spatial distribution.

In this regard, Griffith's SF approach works differently from Getis' (1990, 1995), which decomposes each involved variable into a spatial and a nonspatial component, and requires

\footnotetext{
9 For a discussion of coding schemes and proximity definitions, see, with regard to the German NUTS-3 case, Patuelli et al. (2010b), and more generally Griffith and Peres-Neto (2006). However, across most definitions for spatial weights matrices, the weights corresponding to element $(i, j)$ are highly positively correlated. The results in spatial filtering hardly depend on the matrix from which the eigenvectors are extracted, thus the choice of the weights matrix is of little importance (see Griffith 2000, Getis and Griffith 2002). This is due to the fact that eigenvectors extracted from one (geographical) matrix can almost surely be generated by a linear combination of eigenvectors extracted from any other (geographical) matrix. For example, the matrix $\mathbf{I}_{N}-\rho\left(\mathbf{W}_{N}+\mathbf{W}_{N}^{\prime}\right)$ and its inverse $\left[\mathbf{I}_{N}-\rho\left(\mathbf{W}_{N}+\mathbf{W}_{N}^{\prime}\right)\right]^{-1}$ have the same eigenvectors, although the first may represent just a weighted average across the direct neighbours, whereas the latter represents an (infinite) distance-decay scheme.
} 
the use of non-negative variables. Moreover, differently from mainstream spatial econometric models, such as spatial lag or spatial error models, which are developed mostly in a linear estimation framework, the SF approach can be applied to any functional form. Additionally, the tools necessary for implementing the technique - eigenvector decomposition and stepwise regression - are available in all statistical software packages.

Griffith (2008) shows that SF not only refers to the unobserved spatial correlation of a variable, but also contributes to the explanation of spatial heterogeneity in the parameters. An equivalent to the parameters of a geographically weighted regression (GWR, Brunsdon, Fotheringham and Charlton 1998) can be computed by introducing interaction terms between the exogenous variables of an equation and the eigenvectors extracted from a spatial weights matrix into a model specification. The possibility to combine the SF approach with a panel estimation framework and with geographically heterogeneous regression parameters (SFGWR) constitutes an additional advantage over existing methods. The next section details the functioning of the SFGWR approach.

\subsection{An Adjustment-Process Spatial Filter}

The parameters $\alpha_{i}$ and $\mu_{i}$ in Equations (2) and (3) can be expected to show spatial heterogeneity, ${ }^{10}$ that is, a pattern in space that may be related to the structure of a spatial weights matrix, and for which they could be tested, for example, by computing these parameters' MI. These spatial patterns can be and preferably should be considered explicitly instead of in the parameter-intensive formulation of heterogeneity given in Equations (2) and (3). We introduce spatial patterns by decomposing the terms $\mathbf{A}_{n}$ and/or $\mathbf{M}_{n}$ into a spatial and a non-spatial part, setting $\mathbf{A}_{n}=\omega \tilde{\mathbf{A}}_{k}+\eta_{n}$ and $\mathbf{M}_{n}=\omega \tilde{\mathbf{M}}_{k}+v_{n}$, where $\omega$ is an $n \times k$ matrix of eigenvectors $\mathbf{E}_{k}$ extracted from the normalized spatial weights matrix given in Equation (4) (Griffith 2003). $\omega$ collects the constant (that is, $\mathbf{l}_{n}$ ) as well, because $\mathrm{l}_{n} / \sqrt{n}$ is also an eigenvector of matrix $\mathbf{W} . \eta_{n}$ and $\mathbf{v}_{n}$ contain only non-spatial patterns within the individual parameters - hence they have zero mean and are orthogonal to the spatial process - and can

\footnotetext{
${ }^{10} \mathrm{By}$ the term spatial heterogeneity we refer to spatial structure in the parameters (i.e., the effects of variables), and by the term spatial correlation to spatial structure in variables. However, these terms are insofar related, as on the one hand, spatial correlation (e.g., in a spatial lag or spatial Durbin model) results in spatially heterogeneous marginal impacts (e.g., see LeSage and Pace 2009, Chapter 2.7), and on the other hand, regression parameters can be considered as moments of (multivariate) distributions (in our case, the parameters $\mu_{i}$ represent the region-specific in-sample expectations of the unemployment rate) which may themselves be used as variables.
} 
thus move to the residuals. As we can substitute both the level and the dynamic adjustment in a process by their spatial counterparts, three alternative specifications to Equation (2) yield:

$$
\begin{gathered}
\mathbf{U}_{t} \mathbf{\iota}_{n}=\mathbf{U}_{t-1} \mathbf{A}_{n}+\omega \tilde{\mathbf{M}}_{k}+\mathrm{v}_{n}+\mathbf{S}_{t}+\varepsilon_{t} ; \\
\mathbf{U}_{t} \mathbf{\iota}_{n}=\left(\mathbf{U}_{t-1} \omega\right) \tilde{A}_{k}+\mathbf{U}_{t-1} \eta_{n}+\mathbf{M}_{n}+\mathbf{S}_{t}+\varepsilon_{t} ; \text { and }, \\
\mathbf{U}_{t} \mathbf{\iota}_{n}=\left(\mathbf{U}_{t-1} \omega\right) \tilde{A}_{k}+\mathbf{U}_{t-1} \eta_{n}+\omega \tilde{\mathbf{M}}_{k}+\mathrm{v}_{n}+\mathbf{S}_{t}+\varepsilon_{t} .
\end{gathered}
$$

Equation (5) is the SF equivalent to the FE panel estimation [see Equation (2)]. In contrast, Equations (6) and (7) show similarities with the SF representation of GWR (Griffith 2008). $\tilde{\alpha}_{1}$, the first element of the parameters vector $\tilde{\mathbf{A}}_{k}$, and the one linked to the constant, estimates the average adjustment speed. The further autoregressive parameters specify regional patterns in the adjustment speed: for example, the parameters for the interaction terms between lagged unemployment and eigenvectors $\mathbf{E}_{1}$ and $\mathbf{E}_{2}$ reflect regional deviations from the average adjustment speed along the cardinal coordinates, similarly to the patterns that the eigenvectors themselves represent for the levels. Similarly, the parameters for the subsequent eigenvector interactions reflect how the above deviations can be attributed to more composite spatial patterns: first global, then regional, and finally local.

The new residuals vector - for example, defined as $\zeta_{t}=\mathbf{U}_{t-1} \eta_{n}+v_{n}+\varepsilon_{t}$ in Equation (7) may exhibit either a panel-specific mean-zero component (a random effect, when $\sigma_{v}^{2}>0$ ), or panel-specific serial correlation in the residuals (when $\sigma_{\eta}^{2}>0$ ). Nonetheless, the orthogonality between the spatial eigenvectors and the non-spatial time-constant component suffices to guarantee orthogonality between the regressors $\left(\mathbf{U}_{t-1} \omega, \omega\right)$ and $\zeta_{t}$; that is, consistency of the estimation of Equations (5), (6) and (7). However, the overall variance of these equations is inflated by the variance of $v_{n}$ and/or $\mathbf{U}_{t-1} \eta_{t}$ with respect to Equation (2).

\subsection{Spatial Regimes}

An alternative approach to studying spatial heterogeneity in parameters is the introduction of explicit spatial regimes that, for example, distinguish between urban and rural economies, or to have one regime for each federal state (covering all districts within a single state). Because discrete schemes - in contrast to continuous parameter heterogeneity - allow results to be interpreted as a structural break (Anselin 1990), a common choice in applied work is to use 
just two regimes: typically, North versus South for Europe (Ertur, Le Gallo and Baumont 2006), or East versus West for Germany. In this paper, we apply a classification of regions by the German Federal Institute for Research on Building, Urban Affairs and Regional Development (Bundesinstitut für Bau-, Stadt- und Raumforschung, BBSR), which identifies nine different degrees of urbanization and agglomeration. ${ }^{11}$ The number of spatial regimes to use is rather heuristic, since the classification of districts is due to population density, and is not directly linked to labour market considerations. The intuition is that cities or agglomerations - which have a different industrial and firm structure, different information channels, and populations with different preferences than rural areas - adjust to shocks differently.

In our analysis, we differentiate the (serial) autoregressive parameters (and seasonal effects) according to $r=9$ discrete spatial regimes, and follow the previous estimation approaches for the region-specific levels (by FE or SF). Thus, let $\mathbf{D}_{\text {class }}$ denote the $n \times r$ matrix that assigns a certain urbanization/agglomeration class to each region. In order to avoid perfect multicollinearity, there is no average autoregressive effect included in the equation system. $\xi_{n}$ is the part of spatial heterogeneity in the autoregressive process that is not covered by the regimes, and that is considered unobservable. Then, the two spatial-regimes specifications are given by:

$$
\begin{gathered}
\mathbf{U}_{t} \mathbf{l}_{n}=\left(\mathbf{U}_{t-1} \mathbf{D}_{\text {class }}\right) \widehat{\mathbf{A}}_{r}+\mathbf{M}_{n}+\mathbf{U}_{t-1} \xi_{n}+\mathbf{S}_{t}+\varepsilon_{t} ; \text { and } \\
\mathbf{U}_{t} \mathbf{l}_{n}=\left(\mathbf{U}_{t-1} \mathbf{D}_{\text {class }}\right) \widehat{\mathbf{A}}_{r}+\omega \tilde{\mathbf{M}}_{k}+\mathbf{U}_{t-1} \xi_{n}+v_{n}+\mathbf{S}_{t}+\varepsilon_{t} .
\end{gathered}
$$

In summary, we present three different approaches to model spatially heterogeneous autoregressive processes: by individual, spatial-filtering, and spatial-regimes parameters. In addition, we can estimate a homogeneous parameter as well, as in a standard dynamic panel. The length of the parameter vector $\tilde{\mathbf{A}}_{k}$ in the $\mathrm{SF}$ autoregressive model is $1<k \leq n$; that is, more parameters need to be estimated than in the homogeneous model (with $\alpha_{i}=\bar{\alpha}$ ) and, typically, much less than in the heterogeneous model of Equation (2). Likewise, the number

\footnotetext{
${ }^{11}$ The nine classes are: (1) central cities in regions with urban agglomerations; (2) highly-urbanized districts in regions with urban agglomerations; (3) urbanized districts in regions with urban agglomerations; (4) rural districts in regions with urban agglomerations; (5) central cities in regions with tendencies towards agglomeration; (6) highly-urbanized districts in regions with tendencies towards agglomeration; (7) rural districts in regions with tendencies towards agglomeration; (8) urbanized districts in regions with rural features; and (9) rural districts in regions with rural features.
} 
of spatial-regimes autoregressive parameters is $1<r \leq n$. Thus, both the SF and the spatialregimes autoregressive models are more parsimonious than the individual model.

Theoretically, all other model components are possible to modulate - deterministic mean and seasonal effects - according to the same four schemes. Instead of considering all 64 possible models, in this paper we analyse only specifications where the deterministic mean is represented by FE or the spatial filter, and with homogeneous versus individual (regionspecific) autoregressive and seasonal effects.

\section{Data}

Germany has shown in the past two decades the emergence of interesting dynamics on its regional labour markets and is therefore, for our purposes, a good case study. Analyses in this paper employ data about German regional unemployment rates, at the NUTS-3 level of geographical aggregation (kreise, denominated 'districts' hereforth). The data are available for all 439 districts, on a quarterly basis, for the years 1996 to $2004 .^{12}$

Summary statistics for the data at hand are presented in TABLE 1 . The table results confirm that high and low (regional) unemployment rates are not randomly distributed across Germany. A first examination of the data suggests an asymmetric distribution, which is skewed toward high unemployment rates (the difference between the median and the third quartile is almost one standard deviation). When inspected spatially, the data show marked spatial autocorrelation (Moran's I (MI) for the districts' average unemployment is 0.878), which is further confirmed by descriptive statistics calculated for macro-regional subsets, and by the map in FIGURE 1a. While the former East Germany shows persistently high unemployment rates (averaging 19.4 per cent) with (apparently) little variation (the first quartile is 17 per cent), the former West Germany shows low-to-moderate rates in the North (Northrhine-Westfalia, Lower Saxony, Schleswig-Holstein, and the city-states of Bremen and Hamburg) and in the South (Bavaria, Baden-Wurttemberg, Hesse, Rhineland-Palatinate, and the Saarland). When differencing the data, one can note that a certain amount of spatial

\footnotetext{
${ }^{12}$ The recently formed East German district of Eisenach (ID 16056) belonged to the Wartburgkreis district (ID 16063) until the end of 1997. Thus, unemployment rates for Eisenach before 1998 are not available, and we set them equal to the ones of Wartburgkreis. Also, in the first quarter of 1996, labour force figures are not available for five East German regions. In order to compute unemployment rates, we set the labour force (the denominator of the rate) equal to the labour force reported in the subsequent four quarters (as it is determined only once per year by micro-census data).
} 
autocorrelation remains $(\mathrm{MI}=0.531)$, suggesting that not only the levels of unemployment, but also the dynamics, are spatially correlated. Again, this feature is evident in FIGURE $1 \mathrm{~b}$. This first finding implies that, when estimating a simple AR(1) panel model, one should expect spatial autocorrelation, as well as group-specific serial correlation, in the residuals.

(a)

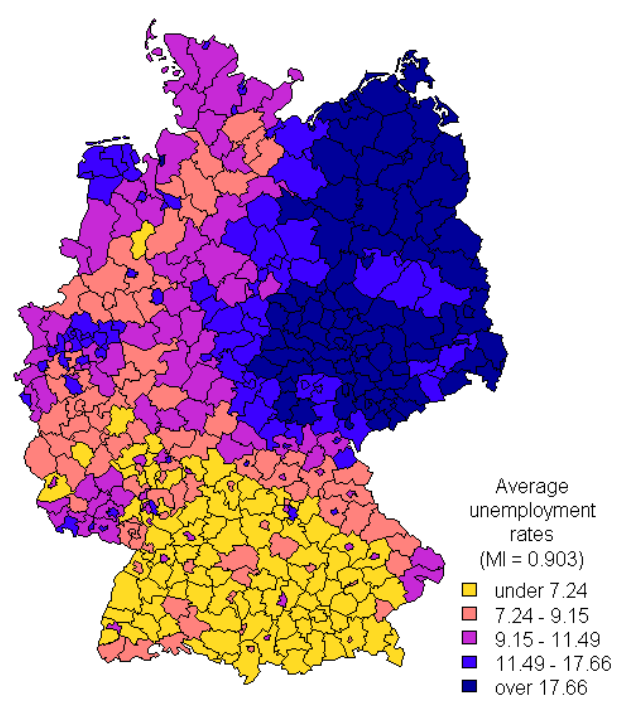

(b)

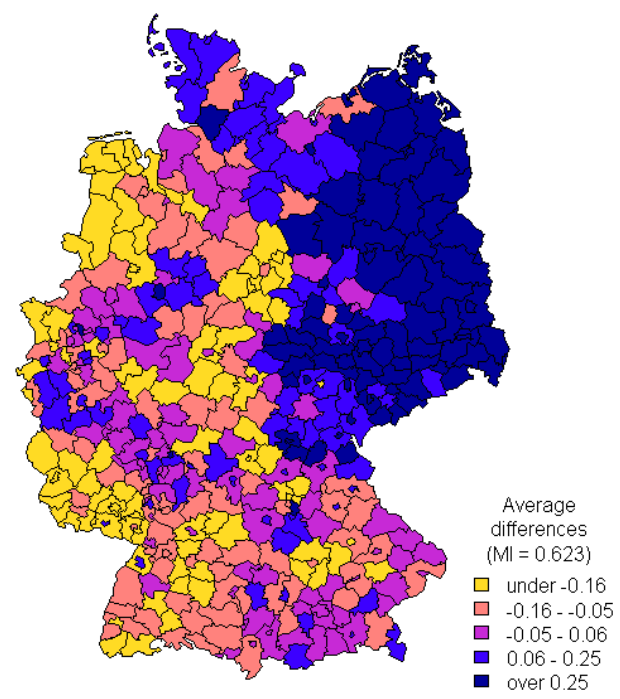

FIGURE 1: Quantile maps of average unemployment rates: in levels (a) and in one-year differences (b).

A further visualization of the data, following Peng (2008), allows a plot of all data $(15,804$ records) simultaneously, providing a bird's eye view over regional disparities and trends. FIGURE 2a shows the unemployment rates of all German districts, by using a common colour scheme, where the different shadings are based on quantiles of the pooled data, and darker shades indicate higher unemployment. The graph (and the accompanying box plots) clearly shows that East German districts (in the bottom rows of each graph) have significantly higher unemployment. Seasonal effects are visible in the background, as the winter quarters show consistently higher unemployment (regularly occurring darker columns). It is also possible to identify some lightly coloured rows among the West German districts (in the left panel roughly at the top quarter, shortly below the first half of the rows for West Germany and little above the thick line separating East and West German districts; these rows indicate heterogeneity in the time-series characteristics within West German local unemployment rates, suggesting the inappropriateness of a homogeneous estimation approach. 

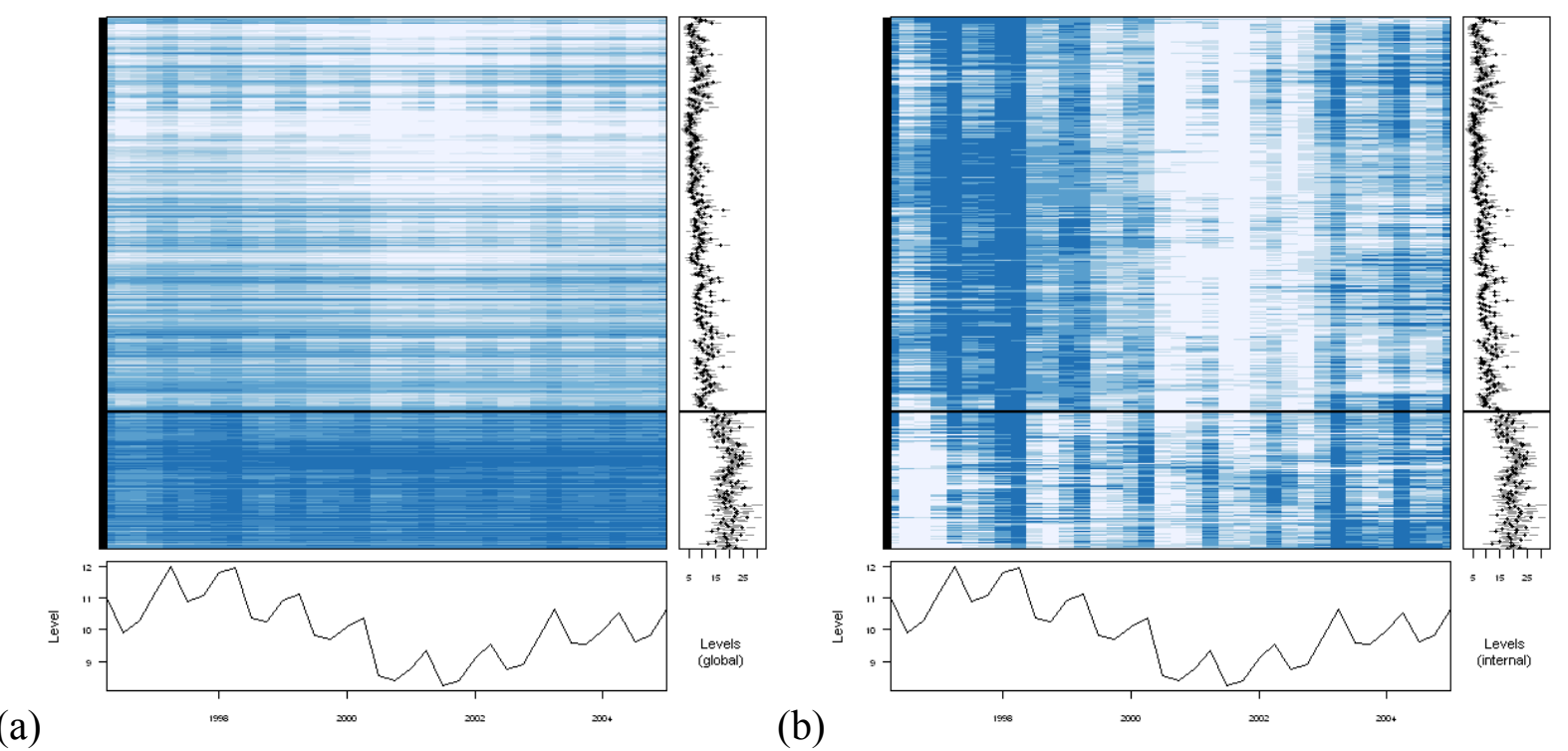

FIGURE 2: Visual representation of German regional unemployment rates.

Note: In the left graph, the colour scheme is common, in right graphs it is region-specific. The thick line separates West German (above) and East German (below) districts. The right margin shows box plots for each district's time series. The bottom margin shows median features.

Assigning to each district its own colour scheme (based on each time series' quantiles), renders FIGURE 2b. Although most West German districts appear to have had their best performance (that is, lowest unemployment rates) between 2000 and 2002, this is not the case for the East German districts. Instead, they seem to have had lower unemployment in $1996 .{ }^{13}$

\section{Empirical Application}

\subsection{Fixed Effects and Spatial Filter Estimation}

In the preceding discussion, we presented a class of dynamic panel models, ranging from standard FE estimation [Equation (2)] to an alternative approach based on surrogating the FE by means of a spatial filter [Equation (5)], to GWR-type spatial filter and spatial regimes models. This subsection presents and compares results obtained for the first (FE and SF) approaches mentioned for a class of models with homogeneous and/or heterogeneous estimates of AR(1) parameters and seasonal effects. In particular, in TABLE 2, we compare

\footnotetext{
${ }^{13}$ In this regard, it should be recalled that no NUTS-3-level unemployment data are available for East Germany before 1996.
} 
summary results such as measures of fit ( $\mathrm{R}^{2}$ and RMSE), (average) autoregressive parameters estimated by the two approaches, and spatial autocorrelation in regression residuals.

TABLE 2: Selected results for the homogeneous and heterogeneous AR process models ${ }^{14}$

\begin{tabular}{|c|c|c|c|c|}
\hline \multirow[t]{2}{*}{ Level } & \multicolumn{2}{|c|}{ Homogeneous seasonality } & \multicolumn{2}{|c|}{ Heterogeneous seasonal effects } \\
\hline & $F E$ & $S F$ & $F E$ & $S F$ \\
\hline \multicolumn{5}{|c|}{ Homogeneous AR(1) process: $\bar{\alpha}$} \\
\hline AR(1) coeff. & 0.766 & 0.945 & 0.901 & 0.957 \\
\hline Av. residuals MI & 0.489 & 0.482 & 0.357 & 0.317 \\
\hline Min. residuals MI & 0.195 & 0.204 & 0.142 & 0.038 \\
\hline Max residuals MI & 0.775 & 0.734 & 0.754 & 0.767 \\
\hline$R^{2}$ & 0.977 & 0.975 & 0.992 & 0.991 \\
\hline RMSE & 0.827 & 0.872 & 0.504 & 0.530 \\
\hline Res. Dfs & 14,922 & 15,321 & 13,608 & 13,979 \\
\hline \multicolumn{5}{|c|}{ Heterogeneous AR(1) process: $\alpha_{i}=A_{n_{i}}$} \\
\hline Av. AR(1) coeff. & 0.833 & 0.823 & 0.906 & 0.914 \\
\hline Min. AR(1) coeff. & $0.135(3462)$ & $0.113(9271)$ & $0.485(14181)$ & $0.594(14188)$ \\
\hline Max. AR(1) coeff. & $1.120(5382)$ & $1.275(5162)$ & $1.035(5711)$ & $1.137(9677)$ \\
\hline No. of $\operatorname{AR}(1) \geq 1$ & $72 / 439$ & $79 / 439$ & $6 / 439$ & $48 / 439$ \\
\hline $\begin{array}{l}\text { No. of AR(1)<1 } \\
\text { (ADF, } 5 \% \text { sign.) }\end{array}$ & $156 / 439$ & $284 / 439$ & $97 / 439$ & $264 / 439$ \\
\hline Av. residuals MI & 0.486 & 0.478 & 0.369 & 0.365 \\
\hline Min. residuals MI & 0.169 & 0.094 & 0.143 & 0.128 \\
\hline Max residuals MI & 0.787 & 0.804 & 0.782 & 0.805 \\
\hline$R^{2}$ & 0.981 & 0.980 & 0.992 & 0.992 \\
\hline RMSE & 0.753 & 0.777 & 0.493 & 0.500 \\
\hline Res. Dfs & 14,484 & 14,865 & 13,170 & 13,564 \\
\hline
\end{tabular}

The top left panel of TABLE 2 compares the most basic model specifications in terms of autoregressive parameters, in which just one (homogeneous) AR(1) parameter is estimated, assuming $\alpha_{1}=\alpha_{2}=\ldots=\alpha_{N}$. The FE and SF approaches are then compared. We find that the computed AR(1) parameters differ between the two approaches. The FE estimation with common seasonal dummies yields a homogeneous $\mathrm{AR}(1)$ parameter of 0.766 , and with region-specific seasonal dummies an $\mathrm{AR}(1)$ parameter of 0.901 . The corresponding (not reported) bias-adjusted parameters - obtained applying a correction according to the formula

\footnotetext{
${ }^{14}$ The (upward biased) autoregressive parameter estimated with a pooled OLS and homogeneous seasonaldummies is 0.993 (with a regionally clustered standard error of 0.0014 ), the asymptotically consistent Blundell-Bond estimator with homogeneous seasonaldummies is 0.902 (with a standard error of 0.0028 ).
} 
for the Nickell bias (see Footnote 5) - would be approximately 0.815 (and 0.955 in case of heterogeneous seasonality). The SF estimations give slightly higher parameters of 0.945 and 0.957, respectively. In anticipation of our further results, the two (corrected) parameter estimates from the FE specifications with homogeneous AR terms are insofar interesting, that they define (approximately) the range in which all other estimates for the average AR parameter fall, that is, the interval running from 0.81 to 0.96 . The difference between the parameters does not seem to be high at first glance. However, the degree of persistence measured as the half-life of a shock given by $\ln 0.5 / \ln \alpha_{i}$ - varies from 3.25 quarters (corresponding to an AR parameter of 0.81 ) to approximately 17 quarters for an $\mathrm{AR}$ parameter of 0.96 .

In terms of model fit, the SF estimate provides a fit to the data - in terms of $\mathrm{R}^{2}-$ very similar to the one for the FE estimate ( 0.975 versus 0.977$)$, while saving about 400 degrees of freedom. As stated in Section 2.3, the variance of the SF estimation is deemed to be (slightly) inflated with respect to the FE variance, which is also suggested by the computation of the RMSE (this is true for all estimations presented in TABLE 2). Meanwhile, in FIGURE 3 we can see how the SF computed (as the linear combination of the 39 eigenvectors selected) approximates the spatial patterns shown in the FE parameters. The spatial patterns shown in the two maps may be expected to include both region-specific variations from the average (homogeneous) AR(1) parameter and seasonal effects, as well as unobserved variables (such as, for example, other lags of the unemployment rate). Not surprisingly, the eigenvector contributing most to the SF is $\mathbf{E}_{2}$, which shows a clear NE-SW pattern, although it should be kept in mind that the amount of variance explained by this top eigenvector, in this dynamic panel framework, is less than 0.7 per cent of the one explained, for example, by the seasonal dummies. Subsequent eigenvectors are at least three times less informative than $\mathbf{E}_{2}$. 
(a)

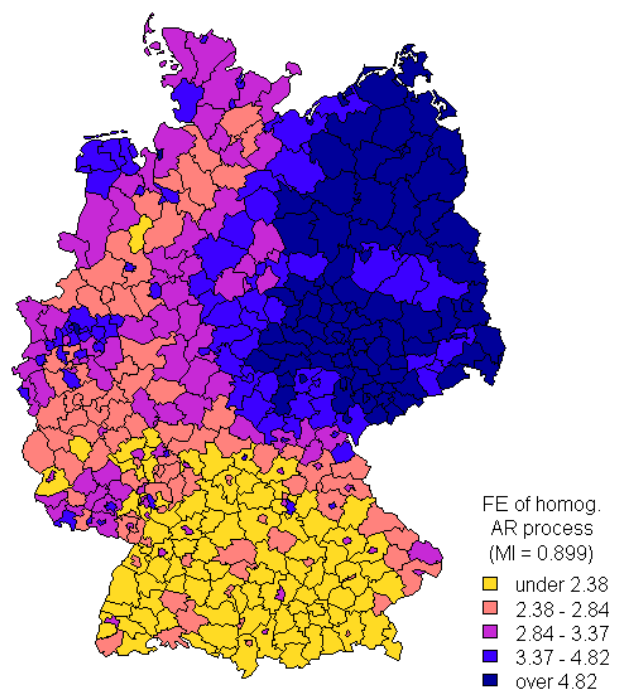

(b)

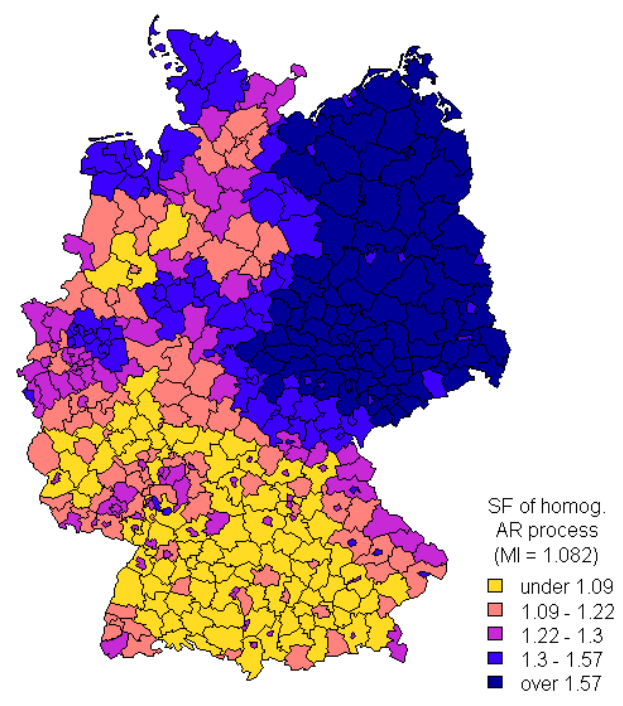

FIGURE 3: Quantile maps of the FE (a) and SF (b) computed for the homogeneous AR(1) process.

Finally, the levels of residual spatial autocorrelation appear to be similar for the FE and SF approaches, with a tendency for the SF approach to obtain residuals slightly less correlated in space. The time-averaged residual per region is zero or very close to zero, and spatial autocorrelation is absent. Consequently, quarter-specific spatial autocorrelation can be related directly to each quarter's specific shocks or unobserved characteristics (beyond direct seasonal effects, which are included in the model), and no recurring pattern exists over time.

Subsequently, the bottom left panel of TABLE 2 provides summary results for estimation of the models presented in Equations (2) and (5), estimating heterogeneous $\operatorname{AR}(1)$ parameters according to the FE and SF approaches, respectively. In contrast with the homogeneous case, where the estimated $\operatorname{AR}(1)$ parameter differed markedly between the two models, the estimates obtained here are rather similar on average, although the number of estimated parameters greater than or equal to 1 is slightly different: 72 and 79 for the FE and SF approaches, respectively. However, tests on the Dickey-Fuller transformation of the system suggest that unit roots can be excluded (at the 95 per cent critical value of a student- $\mathrm{t}$ distribution) for 156 districts in the FE approach and for 284 districts in the SF approach.

Once again, eigenvector $\mathbf{E}_{2}$ is the most informative one, but in this occasion also eigenvector $\mathbf{E}_{1}$ emerges amongst the main ones. The quantity of variance explained by the top eigenvector $\left(\mathbf{E}_{2}\right)$ is now greater in relative terms, for example if compared to the one of the seasonal dummies ( 4 per cent rather than the previous 0.7 per cent). 
A certain level of numerical differences may be expected between the two vectors of AR(1) parameters (given in FIGURE 4). Indeed, the number of eigenvectors selected is distinct between a direct extraction of the SF (the procedure followed in this paper) and an indirect procedure, where FE are computed first, and an SF is extracted from the FE parameters vector. In the former case, fewer eigenvectors are selected, most likely because of the error component $\varepsilon_{t}$ [see Equation (2)] not being considered in the indirect procedure. In contrast, a number of eigenvectors are selected only in the direct procedure, suggesting a correlation between these eigenvectors and the covariates (for example, $\mathbf{U}_{t-1}$ is not assumed to be orthogonal to the eigenvectors). Consequently, possible differences exist between the AR(1) vectors of parameters for Equations (2) and (5). The extent of these differences depends on each specific case, and their direction remains to be fully inspected with a simulation experiment. With regard to the present analysis, clear differences appear to be mostly in the extremes, as shown by the similar quantiles and geographical patterns appearing in FIGURE 4. Both maps indicate higher first-quarter autoregressive effects in the western urbanized areas going (South to North) from Munich to the Stuttgart and Mannheim areas, to the Ruhr and Rhine areas, to Bremen, patterns that generally resemble the spatial distribution of population density in Germany.

(a)

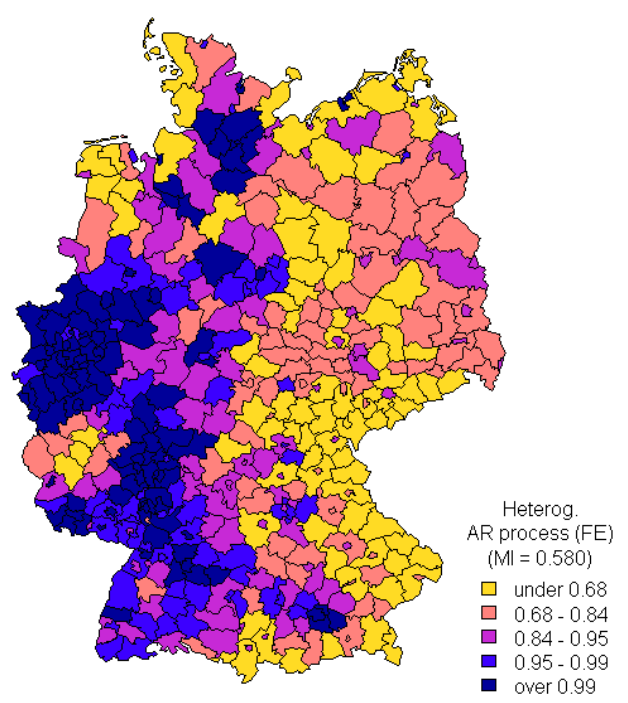

(b)

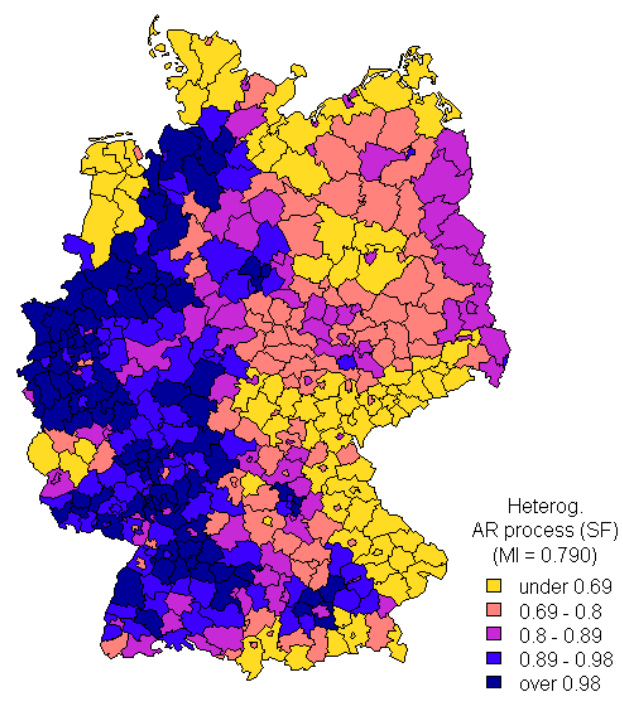

FIGURE 4: Quantile maps of estimated heterogeneous AR(1) parameters: FE (a) and SF (b) approaches [parameters $\alpha_{i}$ according to Equations (2) and (5)].

Conceivably, once we let the autoregressive parameter vary over the cross-section of districts, the measures of fit of the models ( $R^{2}$ and RMSE) improve, while 438 (that is, $n-1$ ) additional degrees of freedom are consumed. Again, the SF estimation allows us to save about 
420 degrees of freedom, while approximating closely the spatial patterns included in the FE parameters (FIGURE 5). Finally, residual spatial autocorrelation is the same - on average - in both the homogeneous and heterogeneous AR(1) parameter estimates, with the SF exhibiting lower minima in this regard.

(a)

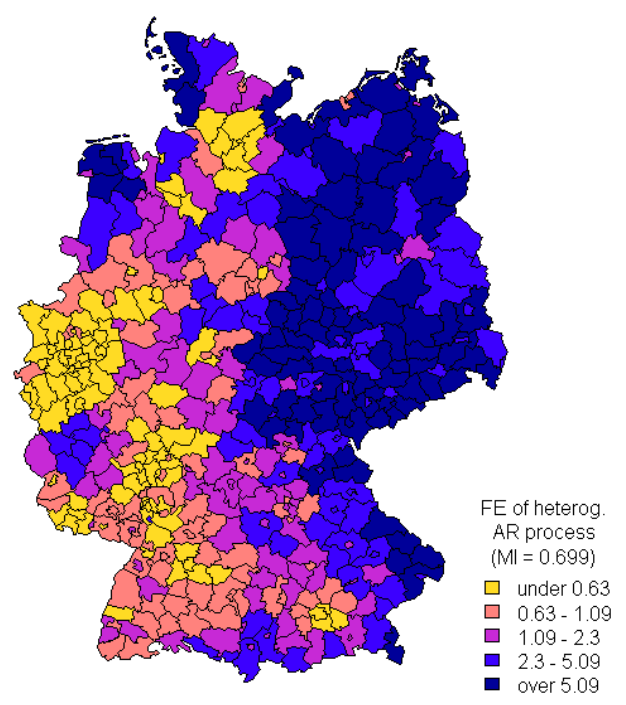

(b)

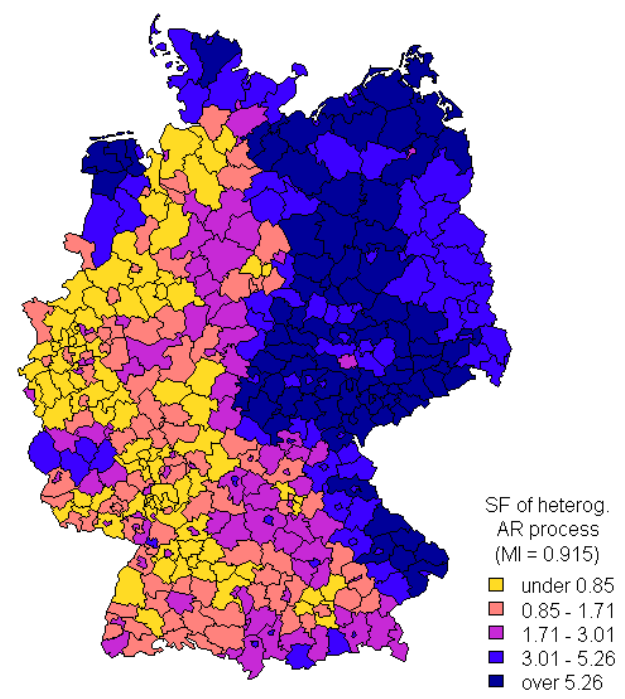

FIGURE 5: Quantile maps of the FE (a) and SF (b) computed for the heterogeneous AR(1) process [levels in Equations (2) and (5)].

Finally, the right-hand panels of TABLE 2 provide additional empirical results, as the above models are extended to include individual (heterogeneous) seasonal effects. This extension implies computing $(439 * 3=)$ 1,317 regression parameters rather than the three previously computed seasonal parameters (for spring, summer and fall, while winter is used as the reference category). In the case in which both the autoregressive and seasonal effects are computed for each district, which we use as our example in the following discussion, (439* 4 $+1=) 1,757$ parameters are computed, which increase to $(439 * 5=) 2,195$ in the FE case. ${ }^{15}$ As a result, an improved fit (higher $\mathrm{R}^{2}$ and lower RMSE) as well as a diminished spatial autocorrelation in the residuals may be expected, which is confirmed by the summary statistics reported in TABLE 2. In addition, higher average AR(1) parameters are found, though with comparable results in terms of unit roots, as suggested by the ADF test results. Noteworthy are the changes in the spatial distribution of the AR(1) parameters and of the FE

\footnotetext{
${ }^{15}$ Needless to say, the increase in computational load leads to a much slower stepwise selection of the SF, which on the other hand may be improved by the use of faster CPUs, by implementing stepwise solutions suitable for multi-core computers or clusters, or by resorting to different types of model selection procedures (see, for example, Miller 2002).
} 
estimates, as shown in FIGURE 6. FIGURE 6a, referring to the AR(1) parameters, portrays patterns appearing in FIGURE 4 that are more sparse, as the result of individual seasonal effects having been filtered out. Meanwhile, FIGURE 6b, appears more similar to FIGURE 5, although it is slightly smoother.

(a)

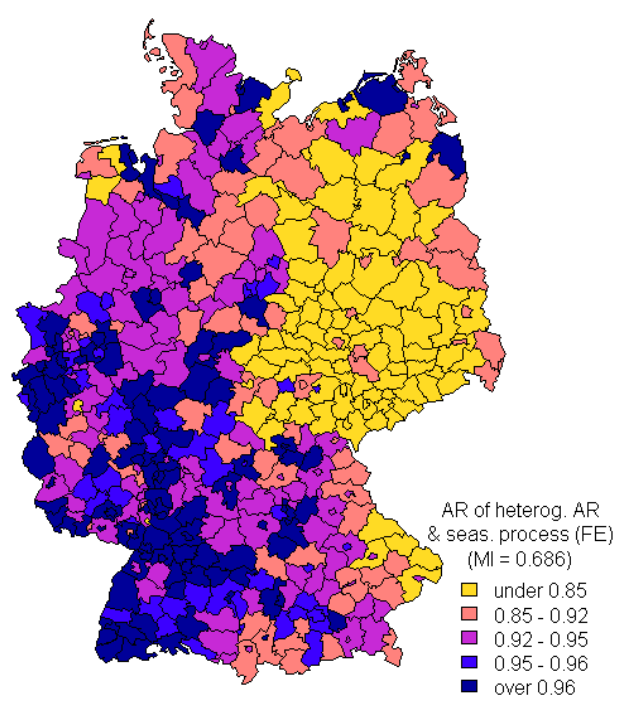

(b)

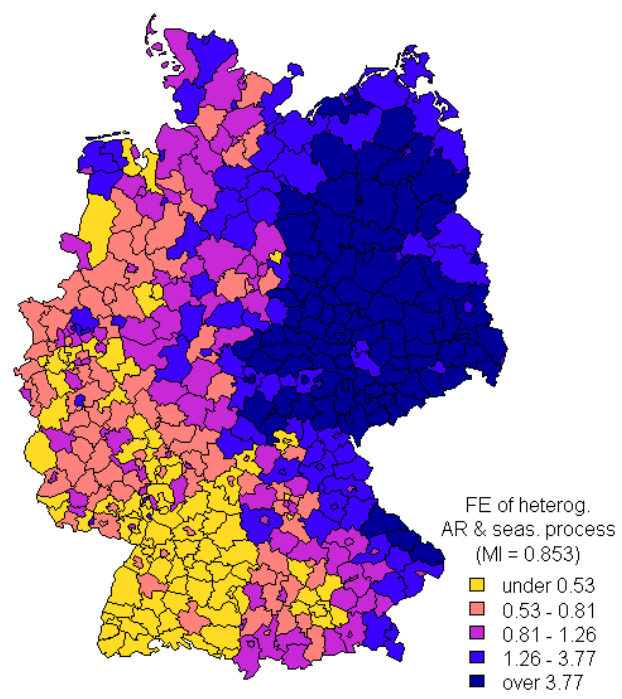

FIGURE 6: Quantile maps of the AR(1) (a) and FE (b) parameters computed for the heterogeneous $\mathrm{AR}(1)$ and seasonal process (FE estimation).

The analyses presented above suggest that SF may be used to approximate the standard FE estimation for the study of unemployment persistence. Each of the two approaches appears to have specific advantages, allowing a researcher to choose freely between them on the basis of his/her needs. However, further approaches to decomposing region-specific autoregressive effects can be employed, as suggested in Sections 2.3 and 2.4. Results obtained for these additional classes of models are presented next.

\subsection{Spatial Filter/Fixed Effects in the Autoregressive Component}

The maps of the AR(1) parameters appearing in FIGURE 4 and the related MI scores highlight that autoregressive parameters are indeed strongly spatially correlated. As proposed in Section 2.3, the spatial patterns obtained according to Equation (5), by computing $n$ autoregressive parameters, may be approximated by parameter expansion in a spatial-filter GWR-fashion. Equations (6) and (7) give the FE and SF specifications, respectively, implying that, for the latter, two spatial filters are computed (or, more generally, one for each SFGWR- 
type regressor, plus the SF substituting the FE). In our specific case, substituting $\mathbf{A}_{n}$ by its SF representation implies saving 392 degrees of freedom (47 versus 439 AR-related regressors), while extending the SFGWR-type approach to seasonal effects allows us to save 1,602 degrees of freedom (154 versus $1,756=439 * 4)$, although at the (opportunity) cost of running extensive stepwise regression in order to select the relevant eigenvectors. ${ }^{16}$ The relevance of such a huge saving in terms of degrees of freedom becomes evident when considering panels with large $N$ and small $T$. In addition, the computational intensity of the spatial filter construction only applies to the first estimation of the model, while subsequent estimations - for example, for forecasting purposes - are faster than in the respective cases of Equations (2) and (5), because the relevant eigenvectors already have been selected.

TABLE 3 reports summary statistics for the aforementioned model specifications. The mean, minimum and maximum AR(1) parameters reported for the SFGWR model (left panel) appear to provide a picture similar to the one found in TABLE 2 for the case of the heterogeneous AR(1) process, with the exception of a higher average parameter in the SF case. The inferential advantage with regard to unit root testing becomes evident: while above the SF model with heterogeneous AR(1) process allows to reject - at a 5 per cent significance level - 264 to 284 unit roots and the FE model with heterogeneous seasonality and AR(1) process has a unit-root rejection rate of less than one quarter of the regions, the SFGWR model leads to a further increase of the rejection rate, reaching 337 unit root rejections for the SFGWR model with heterogeneous seasonality and fixed effects (third column of TABLE 3). ${ }^{17}$ Additionally, we can observe that the GWR models using FEs have roughly the same rejection frequency as the models using SF for the levels (274 vs 270, 337 vs 317) although the estimated average adjustment parameters are smaller in value - that is, the models using SF for the levels seem to be more efficient.

\footnotetext{
${ }^{16}$ Given our starting set of 98 candidate eigenvectors, a backward stepwise regression identifying a SFGWR representation of both the AR(1) parameters and the seasonal effects evaluates, in the first step, $(98 * 4=) 392$ models in the FE case, and $(98 * 5=) 490$ models in the SF case.

${ }^{17}$ For the GWR-type models, the vector of AR(1) parameters is obtained as the linear combination of the related eigenvectors, using as weights the regression parameters computed for the interactions terms between the lagged unemployment rates and the eigenvectors themselves $\left(\alpha_{i}=\omega_{i} \cdot \tilde{\mathbf{A}}_{k}\right)$. Seasonal parameters for each season, when included, are computed in a similar fashion. Because of this construction, unit root tests are computed as $t$-tests, where the variance of each region's autoregressive parameter $\alpha_{i}$ is computed as $\operatorname{var}\left(\alpha_{i}\right)=\sum_{k} \omega_{k i}^{2} \sigma_{k}^{2}$, and $\sigma_{k}^{2}$ is the $k$ th diagonal element of the variance-covariance (sub)matrix of the $K$ eigenvectors selected.
} 
TABLE 3: Selected results for the spatial-filter-GWR (SFGWR) AR process models

\begin{tabular}{|c|c|c|c|c|}
\hline \multirow[t]{2}{*}{ Level } & \multicolumn{2}{|c|}{ Heterogeneous AR(1) process } & \multicolumn{2}{|c|}{$\begin{array}{l}\text { Heterogeneous AR(1) proces } \\
\text { \& seasonal effects }\end{array}$} \\
\hline & $F E$ & $S F$ & $F E$ & $S F$ \\
\hline \multicolumn{5}{|c|}{ Spatial filter AR(1) process: $\alpha_{i}=\omega_{i} \cdot \tilde{\mathbf{A}}_{k}$} \\
\hline Av. AR(1) coeff. & 0.853 & 0.935 & 0.882 & 0.961 \\
\hline Min. AR(1) coeff. & $0.162(9276)$ & $0.276(9271)$ & $0.530(14188)$ & $0.697(9271)$ \\
\hline Max. AR(1) coeff. & $1.238(7338)$ & $1.211(5374)$ & $1.163(9274)$ & $1.140(5374)$ \\
\hline No. of $\operatorname{AR}(1)>1$ & $94 / 439$ & $136 / 439$ & $44 / 439$ & $94 / 439$ \\
\hline No. of $\operatorname{AR}(1)<1$ & $274 / 439$ & $270 / 439$ & $337 / 439$ & $317 / 439$ \\
\hline \multicolumn{5}{|l|}{ (ADF, 5\% sign.) } \\
\hline Av. residuals MI & 0.481 & 0.440 & 0.333 & 0.176 \\
\hline Min. residuals MI & 0.139 & 0.129 & 0.012 & -0.016 \\
\hline Max residuals MI & 0.817 & 0.730 & 0.803 & 0.704 \\
\hline $\mathrm{R}^{2}$ & 0.980 & 0.978 & 0.985 & 0.986 \\
\hline RMSE & 0.776 & 0.824 & 0.666 & 0.650 \\
\hline Res. Dfs & 14,876 & 15,227 & 14,772 & 15,064 \\
\hline Selected eigenvecs & 46 & 64 & 27 & 46 \\
\hline for SFGWR-AR(1) & & & & \\
\hline
\end{tabular}

Once again, the levels of spatial autocorrelation in the residuals vary greatly, depending on quarter-specific noise, and are comparable but slightly lower than the earlier ones. RMSE increases moderately, as expected, but is being balanced out by the aforementioned huge savings in terms of degrees of freedom. These results are confirmed by extending the SFGWR specification to seasonal effects (right panel).

In terms of the spatial autocorrelation observed in the AR(1) parameters resulting from Equations (6) and (7), FIGURE 7 confirms the similarities with the spatial distribution of population density. The spatial distribution of the estimated FE and SF (plotted in FIGURE 8) again is consistent pairwise, showing higher unexplained variation in the levels for East German districts. Not surprisingly, the light-shaded areas of FIGURE 7 appear to match the dark-shaded areas of FIGURE 8, as greater relative stability in the East German unemployment rates due to time-constant unobserved regional characteristics (or just lower dependence from their one-quarter lag) is reflected in the FE or in the SF. Similar observations can be made by comparing FIGURE 4 and 6, or the two maps in FIGURE 6. 
(a)

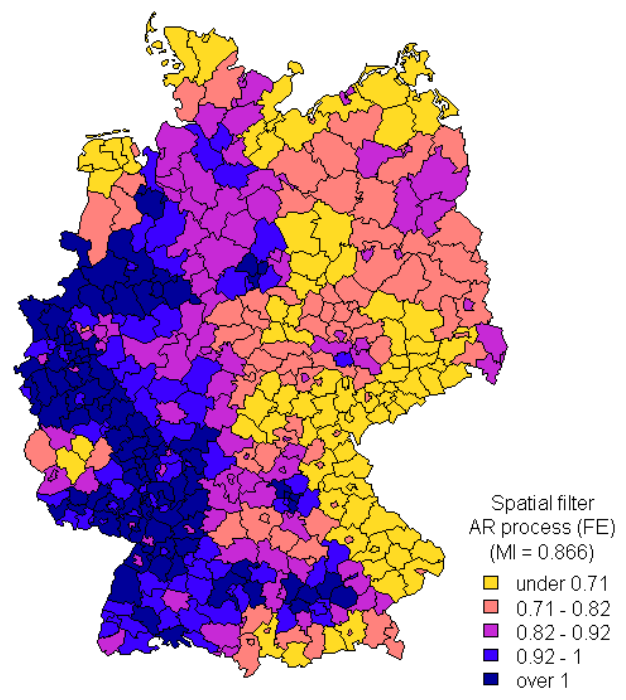

(b)

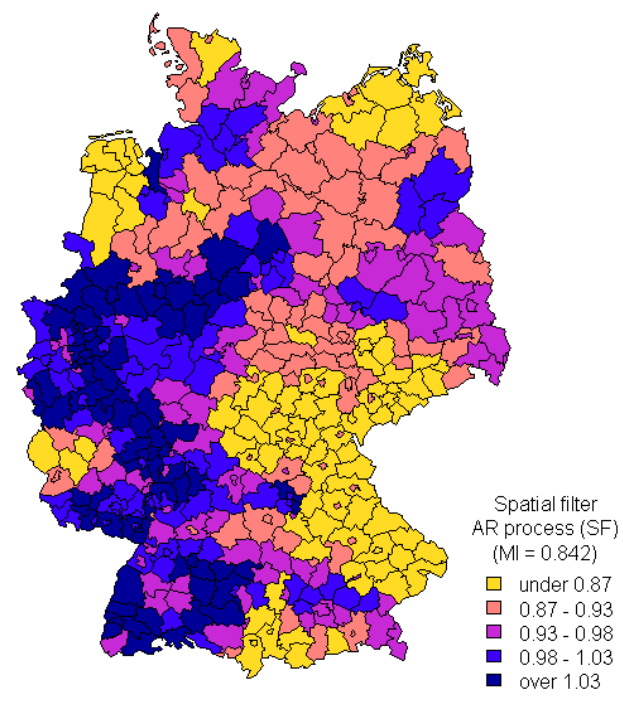

FIGURE 7: Quantile maps of estimated spatial-filter-GWR (SFGWR) AR(1) parameters: FE (a) and SF (b) approaches.

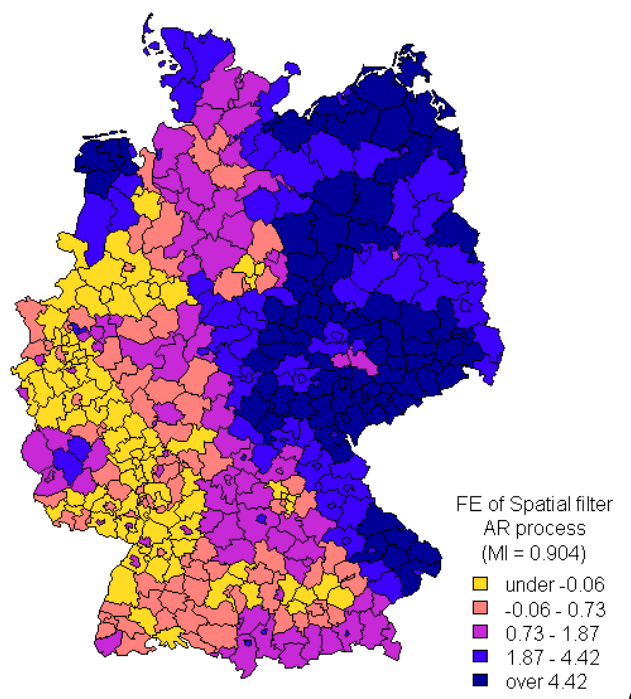

(b)

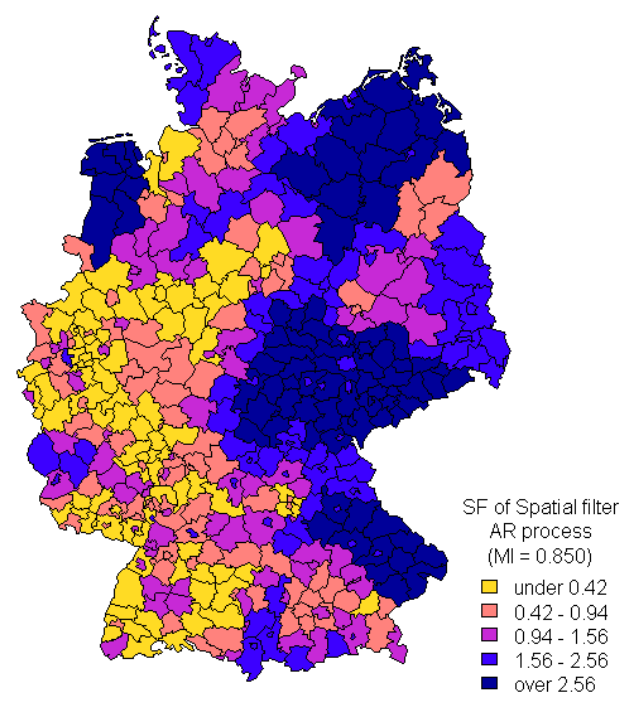

(a)

FIGURE 8: Quantile maps of the FE (a) and SF (b) computed for the spatial filter AR(1) process.

As we already noted, the spatial-filter GWR surrogate for the region-specific autoregressive parameters allows identification of the spatial structure underlying the heterogeneity of the dynamic labour market process. Amongst the selected eigenvectors in the SFGWR specification with a spatial filter for the level component and homogeneous seasonal figures (FIGURE $7 \mathrm{~b}$ and FIGURE 8b), there are four (of the five) eigenvectors associated with global 
patterns - that is, eigenvectors which, when the values are plotted into a map, show one or two large 'peaks' and one or two big 'valleys' spreading out over a large areas. 40 selected eigenvectors can be associated with regional, and 20 with local patterns. ${ }^{18}$ Since all eigenvectors have the same scale (their values have an identical standard deviation), the partial contribution of each eigenvector to the overall autoregressive process is sized proportionately to the absolute value of the corresponding parameter. However, amongst the 15 eigenvectors with the highest parameter in absolute value, only two are global and two are local (the first local is at position 13), but 11 eigenvectors reflect regional patterns. In the other specifications, we find a similar selection of eigenvectors (the same four global, and roughly twice as many regional as local). However, in the corresponding SFGWR estimation using fixed effects (i.e., when the levels are forced to show maximum heterogeneity), all four global eigenvectors are amongst the 15 most influential eigenvectors.

More interestingly, there is a negative relation between the parameters associated with the (common) eigenvectors selected for modelling serial dependence and for the levels, as suggested by FIGURE 9. Additionally, eigenvectors which are selected only in one case (for which we include a value of zero in case of non-selection) have parameter values closer to zero even when significant, showing that the common eigenvectors are the ones with the greatest importance in both filters. On the other hand, the negative Pearson correlation of 0.89 ( -0.93 for the common subset) between the two sets of parameters suggests that the SF in the levels behaves in the opposite way than the SF for the AR(1) parameters. ${ }^{19}$ This indicates a trade-off between the level of persistence (i.e., serial dependence) and the influence of the (deterministic) level showing the spatial pattern modelled by the filter: unemployment is then represented as a weighted average of (more or less) persistent random elements (with a set of weight $a$ ) and deterministic elements [with weights $(1-a)$ ] The more unemployment in a certain number of contingent regions (described by the mapping pattern of the eigenvectors) is driven by persistent shocks, the less important are the deterministic components in these regions - and vice versa, the lower the persistence, the faster regions adjust towards their initial (or natural) levels which become more important. This finding calls for further analytical investigation, which goes beyond this paper's objective.

\footnotetext{
${ }^{18}$ The classification of global, regional and local eigenvectors is according to the table for 98 candidate eigenvectors extracted from a rook C-coding matrix given by Patuelli et al. (2010b). Eigenvectors 1 to 5 are considered global, 6 to 66 regional and 67 to 98 local.

${ }^{19}$ A similar finding is obtained when both the AR(1) and the seasonal parameters are computed by means of the GWR-SF approximation. A Pearson correlation of -0.83 is obtained the two sets, and -0.91 is found for the common sets.
} 


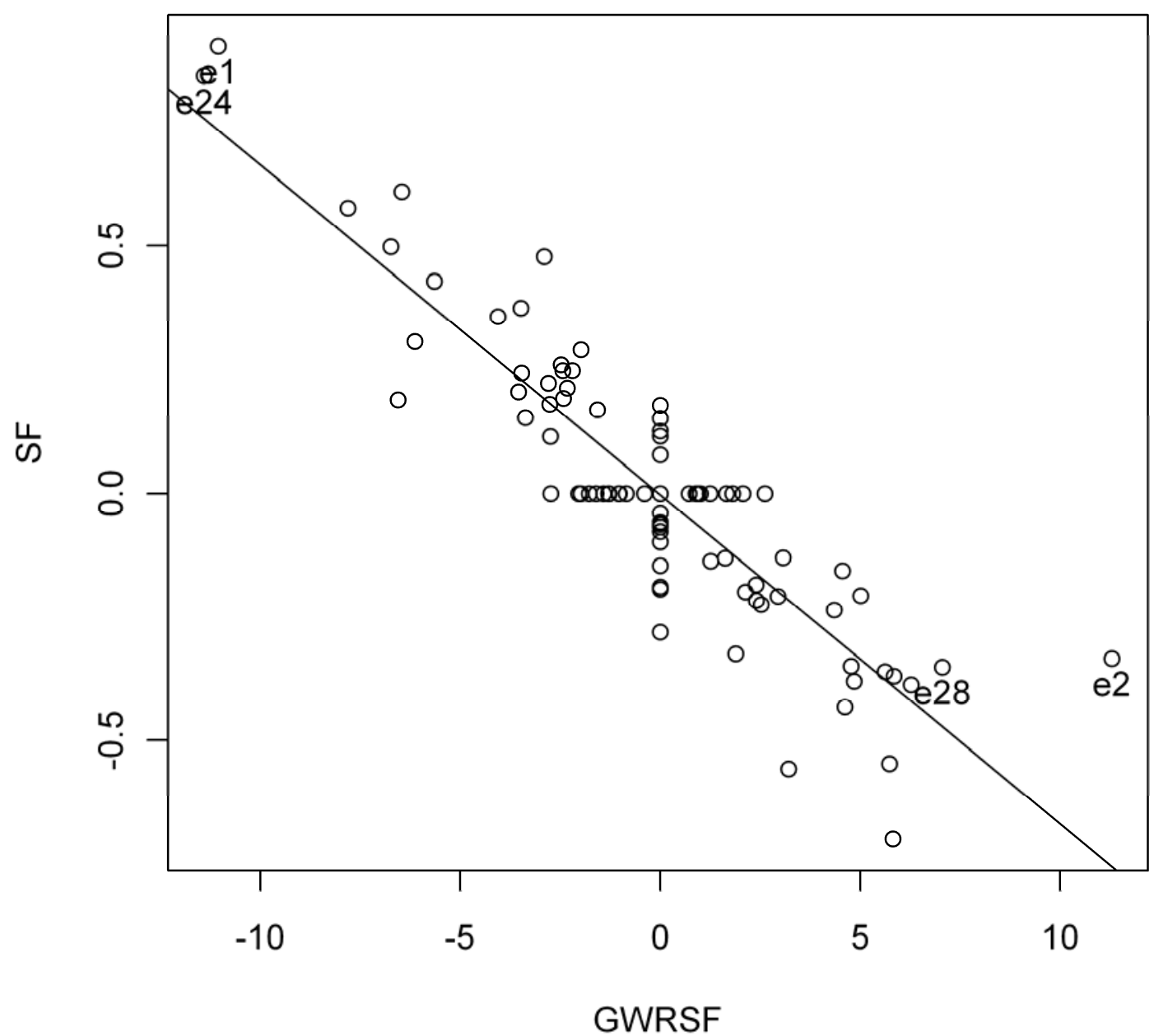

FIGURE 9: Correlation between the parameters of the eigenvectors selected for the SFGWR AR interpolation and for the levels (with homogeneous seasonal effects).

Finally, the residual variance and the number of parameters of the models presented above can be combined to compute various information criteria (see TABLE 5, in the Annex). The Akaike information criterion (AIC) suggests that the SFGWR specification for the autoregressive process uses the information best, when compared to other model specifications, and that FE in the levels are superior to the SF. However, the AIC is often considered not adequate (or weak) for finite samples, and other criteria may be more reliable. The Schwartz Bayesian information criterion (BIC), which is often found to be over-selective, indicates superiority of the SF in the levels compared to the FE, and superiority of the SF AR process as well, because of the greater importance given to the degrees of freedom saved. The 
advantage of spatial filters in modelling both levels and autoregressive processes is confirmed by the Hannan-Quinn information criterion (HQ).

\subsection{Adjustment to Shocks According to the Spatial Regimes}

In our final analysis, we present, in TABLE 4, summary statistics for the spatial regimes specification introduced in Equations (8) and (9). In these specifications, heterogeneity of the autoregressive parameters is introduced by distinguishing between districts with different levels of agglomeration and urbanization. Consequently, instead of $n$ AR(1) parameters, only nine are computed, corresponding to the specific classes introduced in Section 2.4. This approach makes identification of (average) autoregressive (and seasonal) effects possible for classes such as city-districts in agglomerated areas, or rural districts belonging to rural areas. The results obtained by applying the spatial regimes decomposition to the AR(1) process alone are shown in the left panel of TABLE 4. We obtain nine AR(1) parameters ranging from 0.613 to 0.984 in the FE case, and from 0.927 to 0.949 in the SF case. These results are consistent with our previous findings (see TABLE 2). It turns out that the average AR parameters are higher for the SF approach, but when employing ADF tests only the FE case presents a unit root. This single unit root (which is not confirmed when decomposing seasonal effects as well) is found for districts of type 1 (that is, 'central cities in regions with urban agglomerations'). Our findings confirm the tendency of the AR(1) parameters to resemble the spatial distribution of population density, and of the central business districts (CBDs) of dense regions to show the highest parameters. FIGURE 10 maps the values found for the spatial regimes $\mathrm{AR}(1)$ parameters (SF estimation with homogeneous seasonal effects), and clearly shows that this approach provides a rough approximation of the parameter estimates obtained above, while showing - within a general picture of high persistence - some core-periphery patterns between the 'central cities' (type 1 and 5 districts, with higher persistence) and their surroundings; equality of all nine AR parameters is rejected both in the FE estimations and in the SF estimations. However, the regimes approach associates also a high degree of persistence to agglomerated areas in Eastern Germany (e.g., Dresden, Berlin or Chemnitz) which has not been found when using individual parameters (see FIGURE 4), that is, this rough approximation may indeed be missing some pattern. There are pros and cons to using spatial regimes, and this preliminary finding may deserve further investigation in the future research. 
TABLE 4: Selected results for the spatial-regimes AR(1) process models

\begin{tabular}{|c|c|c|c|c|}
\hline \multirow[t]{2}{*}{ Level } & \multicolumn{2}{|c|}{ Heterogeneous AR(1) process } & \multicolumn{2}{|c|}{$\begin{array}{l}\text { Heterogeneous } A R(1) \text { process \& } \\
\text { seasonal effects }\end{array}$} \\
\hline & $F E$ & $S F$ & $F E$ & $S F$ \\
\hline \multicolumn{5}{|c|}{ Spatial-regimes $\operatorname{AR}(1)$ process: $\alpha_{i}=\mathbf{D}_{i} \cdot \widehat{\mathbf{A}}_{r}$} \\
\hline Av. AR(1) coeff. & 0.808 & 0.937 & 0.812 & 0.946 \\
\hline Min. AR(1) coeff. & 0.613 (type 9) & 0.927 (type 9) & 0.670 (type 3 ) & 0.916 (type 2$)$ \\
\hline Max. AR(1) coeff. & $0.984($ type 1$)$ & 0.949 (type 5) & 0.934 (type 1$)$ & 0.960 (type 9$)$ \\
\hline No. of $\operatorname{AR}(1) \geq 1$ & $0 / 9$ & $0 / 9$ & $0 / 9$ & $0 / 9$ \\
\hline $\begin{array}{l}\text { No. of } \mathrm{AR}(1)<1 \\
\text { (ADF, } 5 \% \text { sign.) }\end{array}$ & $8 / 9$ & $9 / 9$ & $9 / 9$ & $9 / 9$ \\
\hline Av. residuals MI & 0.485 & 0.476 & 0.425 & 0.417 \\
\hline Min. residuals MI & 0.195 & 0.198 & 0.167 & 0.178 \\
\hline Max residuals MI & 0.769 & 0.746 & 0.747 & 0.729 \\
\hline$R^{2}$ & 0.978 & 0.975 & 0.981 & 0.979 \\
\hline RMSE & 0.810 & 0.869 & 0.754 & 0.798 \\
\hline Res. Dfs & 14,914 & 15,306 & 14,890 & 15,291 \\
\hline
\end{tabular}

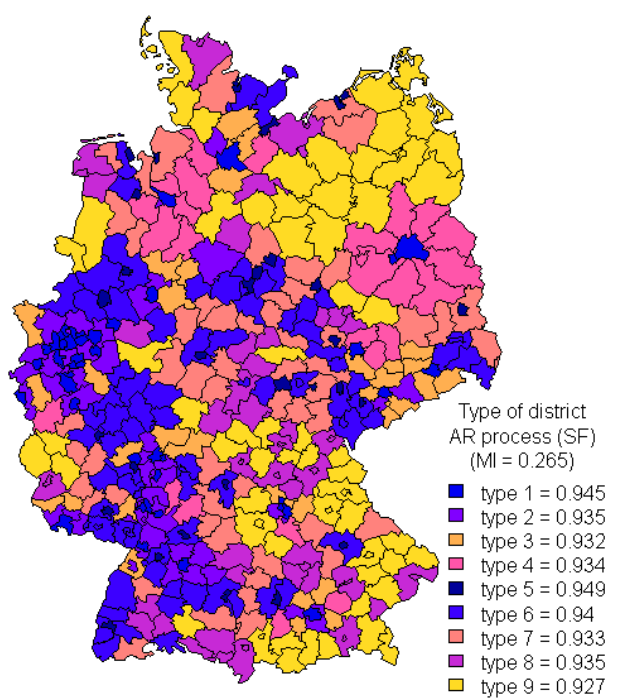

FIGURE 10: Map of estimated spatial-regimes AR(1) parameters: SF approach [parameters $\alpha_{r}$ according to Equation (9)].

\subsection{Concluding Remarks: Persistence of Unemployment}

The empirical findings presented in this section give a clear picture of unemployment persistence in Germany. We find the adjustment speed of regional unemployment to shocks to 
be extremely heterogeneous, which makes estimation of a single AR-parameter look unreasonable and supports our call for regionally disaggregated estimations. Modelling the heterogeneity by SF-GWR seems to capture most of this heterogeneity, but spatial regimes do surprisingly well too. The averages over the AR parameters - and the majority of them throughout the various specifications lie between 0.76 and 0.96 , that is, close to 1 . Thus, shocks to unemployment may be expected to be persistent, or at least to have a long half-life in most regions. For example, an AR parameter of 0.8 is equivalent to a half-life of more than three quarters, or the effect of the shock vanishing after eight years (10 times the half-life); an AR parameter of 0.9 corresponds to a half-life of 6.6 quarters, and a parameter of 0.95 to a half-life of 13.5 quarters. When using Dickey-Fuller equivalent transformations of the models, we can reject the hypothesis that the difference of the average autoregressive parameter minus one - the average of this distance is between -0.24 and -0.04 - is greater than or equal to zero. At least on average, unemployment is stationary - a necessary condition for the existence of (conditional) convergence - although non-stationarity can hardly be rejected for a large fraction of regions. Thus, unemployment adjusts very slowly - if ever - toward a kind of natural rate; it behaves (in particular in the agglomerated districts along the river Rhine) more like a random walk. Saying that there is clear evidence of (cross-sectional) convergence among the rates would be an excessive statement.

Our findings are particularly significant with regard to exogenous shocks: positive, in the case of active labour market policy interventions; negative, as in the case of the recent global economic crisis. Strong persistence of the regional unemployment rates suggests that a negative shock, due for example to a sudden increase in labour supply, to not-anticipated deflation, or to economic catastrophes, would take a rather long time to be absorbed. We can think, for example, of new labour regulations for foreign workers (the enlargement of the European Union from EU-15 to EU-25), of the collapse of the states/markets belonging to the socialist Council for Mutual Economic Aid (Comecon) in the late 1980s/early 1990s (affecting the former German Democratic Republic), or of political events as in Card (1990). In this regard, there is potential in expanding the above analyses to the analysis of relative unemployment, which appears to have different persistence dynamics than the absolute levels [see, e.g., Jimeno and Bentolila (1998), where the determinants of unemployment persistence are also discussed]. 


\section{Conclusions}

Studies about the convergence or persistence of unemployment typically employ univariate autoregressive equations and test them for stationarity. This procedure is straightforward and computationally simple, but can hardly account for cross-sectional heterogeneity and dependence - thus, in the best case, it is statistically inefficient (imprecise) or, in the worst case, mispecified. Derived conclusions may then be misleading.

In this paper, we have focused on two questions. First, starting with a system of AR(1) equations, we aimed to show the substitutability of fixed effects (FE) and spatial filters and, analogously for autoregressive processes, the one between individual autoregressive parameters and SF GWR-type estimation. The SF surrogates [which allow to decompose the FE into a spatially structured and a spatially unstructured (random) part] are more parsimonious with regard to the number of parameters, and use, instead of region-specific parameters, a set of parameters defined and computed over all regions.

Second, we applied SF methods when analysing the dynamics of quarterly regional unemployment rates for Germany from 1996 to 2004. Because the eigenvectors employed in an SF represent map patterns, one advantage of this approach is that the heterogeneous autoregressive adjustment parameters of the GWR-type models have a geographical interpretation. For comparison, we also provided estimates of a homogeneous autoregressive process, and of one approach differentiated according to nine urbanization/agglomeration regimes.

Indeed, when comparing pairwise the individual and SF specifications for the process component (AR or level), keeping everything else equal, we found that the SF approach provides a gain in residual degrees of freedom, without losing much estimation accuracy, measured, for example, in terms of goodness-of-fit $\left(R^{2}\right)$ or root mean squared error (RMSE). We found, for the SF AR specification, some gain in precision when compared with the homogeneous and spatial regime specifications. Summary diagnostics for all models, based on information criteria, provided a confirmation of the potential of the proposed SF-based models. The residuals from individually-specified models and of their corresponding SF equivalents are highly correlated, and the error distributions are quite similar pairwise. The estimates for the average autoregressive parameter vary, in particular, between the FE estimation with homogeneous seasonal effects (0.76-0.85) and the remaining level/seasonality combinations (0.90-0.96). Consequently, a potential bias in the autoregressive parameter does not seem to depend on the way in which the autoregressive 
process is specified. However, obtaining exact evidence about the consistency of the AR estimates is only possible by means of Monte Carlo simulation. This aspect will be the subject of future research, since here we limit ourselves to showcasing the practical relevance of the proposed approaches. A further aspect that may be expected to be investigated in future research is the extension of the proposed models to the estimation of nonlinear regression models (e.g., in the case of unemployment rates, the logistic regression), for which panel models are generally less popular in the econometric literature and competition with other applied statistics fields is stronger (e.g., generalized linear mixed models).

\section{Acknowledgments}

The first author wishes to thank Paolo Foschi and Simone Giannerini (University of Bologna, Italy), as well as Cristian Bianchi, Alberto Ferrante and Alex Villazon (University of Lugano, Switzerland), for their help in executing the computational problems encountered during the preparation of this paper. We also wish to thank, for useful comments and suggestions, the Editor, three anonymous referees, seminar participants at VU University Amsterdam and the University of Lugano, and session participants to the 55th Annual North American Meetings of the Regional Science Association International (New York, NY), the 49th European Congress of the Regional Science Association International (Lódź), the ZEW/IAB Workshop on Spatial Dimensions of the Labour Market (Mannheim), the SSES Annual Meeting 2010 (Fribourg), and the 16th International Conference on Panel Data (Amsterdam). 


\section{References}

Aldashev, Alisher. 2009. "Occupational and Locational Substitution: Measuring the Effect of Occupational and Regional Mobility," ZEW Discussion Paper No. 09-014, ZEW.

Anselin, Luc. 1988. Spatial Econometrics: Methods and Models. Dordrecht Boston: Kluwer Academic Publishers.

. 1990. "Spatial Dependence and Spatial Structural Instability in Applied Regression Analysis," Journal of Regional Science, 30, no. 2: 185-207.

Aragon, Yves, Dominique Haughton, Jonathan Haughton, Eve Leconte, Eric Malin, Anne Ruiz-Gazen, and Christine Thomas-Agnan. 2003. "Explaining the pattern of regional unemployment: the case of the Midi-Pyrénées region," Papers in Regional Science, 82, no. 2: $155-174$.

Armstrong, Harvey, and Jim Taylor. 2000. Regional Economics and Policy. 3rd ed. Oxford: Blackwell.

Badinger, Harald, and Thomas Url. 2002. "Determinants of Regional Unemployment: Some Evidence from Austria," Regional Studies, 36, no. 9: 977-988.

Basile, Roberto, and Luca De Benedictis. 2008. "Regional Unemployment and Productivity in Europe," Papers in Regional Science, 87, no. 2: 173-192.

Bayer, Christian, and Falko Juessen. 2007. "Convergence in West German Regional Unemployment Rates," The German Economic Review, 8, no. 4: 510-535.

Blanchard, Olivier J., and Lawrence H. Summers. 1986. "Hysteresis and the European Unemployment Problem," NBER Macroeconomics Annual, 1: 15-78.

Blundell, Richard, and Stephen Bond. 1998. "Initial Conditions and Moment Restrictions in Dynamic Panel Data Models," Journal of Econometrics, 87, no. 1: 115-143.

Brunsdon, C, S Fotheringham, and M Charlton. 1998. "Geographically Weighted Regression," Journal of the Royal Statistical Society: Series D (The Statistician), 47, no. 3: 431-443.

Bun, Maurice J. G., and Martin A. Carree. 2005. "Bias-Corrected Estimation in Dynamic Panel Data Models," Journal of Business and Economic Statistics, 23, no. 2: 200-210.

Card, David. 1990. "The Impact of the Mariel Boatlift on the Miami Labor Market," Industrial and Labor Relations Review, 43, no. 2: 245-257.

Cracolici, Maria Francesca, Miranda Cuffaro, and Peter Nijkamp. 2007. "Geographical Distribution of Unemployment: An Analysis of Provincial Differences in Italy," Growth and Change, 38, no. 4: 649-670.

Decressin, Jorg, and Antonio Fatás. 1995. "Regional Labour Market Dynamics in Europe," European Economic Review, 39, no. 9: 1627-1655.

Elhorst, J. Paul. 1995. "Unemployment Disparities between Regions in the European Union," in H. W. Armstrong and R. W. Vickerman (eds.), Convergence and Divergence among European Unions. London: Pion, pp. 209-221.

2003. "The Mystery of Regional Unemployment Differentials: Theoretical and Empirical Explanations," Journal of Economic Surveys, 17, no. 5: 709-748.

Ertur, Cem, and Julie Le Gallo. 2003. "An Exploratory Spatial Data Analysis of European Regional Disparities, 1980-1995," in B. Fingleton (ed.), European Regional Growth. Berlin Heidelberg New York: Springer-Verlag, pp. 55-98.

Ertur, Cem, Julie Le Gallo, and Catherine Baumont. 2006. "The European Regional Convergence Process, 1980-1995: Do Spatial Regimes and Spatial Dependence Matter?," International Regional Science Review, 29, no. 1: 3-34. 
Fuchs, Johann, Markus Hummel, Sabine Klinger, Eugen Spitznagel, Susanne Wanger, and Gerd Zika. 2010. "Der Arbeitsmarkt schließt an den vorherigen Aufschwung an," IAB Kurzbericht No. 18/2010, IAB.

Garcia-del-Barrio, Pedro, and Luis Alberiko Gil-Alana. 2009. "New Revelations About Unemployment Persistence in Spain: Time-Series and Panel Data Approaches Using Regional Data," Applied Economics, 41, no. 2: 219 - 236.

Getis, Arthur. 1990. "Screening for Spatial Dependence in Regression Analysis," Papers of the Regional Science Association, 69: 69-81.

. 1995. "Spatial Filtering in a Regression Framework: Examples Using Data on Urban Crime, Regional Inequality, and Government Expenditures," in L. Anselin and R. J. G. M. Florax (eds.), New Directions in Spatial Econometrics. Heidelberg: Springer, pp. 172-185.

Getis, Authur, and Daniel A. Griffith. 2002. "Comparative Spatial Filtering in Regression Analysis," Geographical Analysis, 34, no. 2: 130-140.

Griffith, Daniel A. 2000. "A Linear Regression Solution to the Spatial Autocorrelation Problem," Journal of Geographical Systems, 2, no. 2: 141-156.

- 2003. Spatial Autocorrelation and Spatial Filtering: Gaining Understanding through Theory and Scientific Visualization. Berlin New York: Springer.

- 2008. "Spatial-Filtering-Based Contributions to a Critique of Geographically Weighted Regression (GWR)," Environment and Planning A, 40, no. 11: 2751-2769.

Griffith, Daniel A., and Pedro R. Peres-Neto. 2006. "Spatial Modeling in Ecology: The Flexibility of Eigenfunction Spatial Analyses," Ecology, 87, no. 10: 2603-2613.

Im, Kyung So, M. Hashem Pesaran, and Yongcheol Shin. 2003. "Testing for Unit Roots in Heterogeneous Panels," Journal of Econometrics, 115, no. 1: 53-74.

Jimeno, Juan F., and Samuel Bentolila. 1998. "Regional Unemployment Persistence (Spain, 1976-1994)," Labour Economics, 5, no. 1: 25-51.

Kochendörfer-Lucius, Gudrun, and Boris Pleskovic, eds., 2009. Spatial Disparities and Development Policy. Washington, DC: World Bank.

Kosfeld, Reinhold, and Christian Dreger. 2006. "Thresholds for Employment and Unemployment. A Spatial Analysis of German Regional Labour Markets 1999-2000," Papers in Regional Science, 85, no. 4: 523-542.

Lee, Chien-Chiang, and Chun-Ping Chang. 2008. "Unemployment Hysteresis in OECD Countries: Centurial Time Series Evidence with Structural Breaks," Economic Modelling, 25, no. 2: 312-325.

LeSage, James P., and R. Kelley Pace. 2009. Introduction to Spatial Econometrics. Boca Raton: CRC Press.

Levin, Andrew, Chien-Fu Lin, and Chia-Shang James Chu. 2002. "Unit Root Tests in Panel Data: Asymptotic and Finite-Sample Properties," Journal of Econometrics, 108, no. 1: $1-24$.

López-Bazo, Enrique, Tomás del Barrio, and Manuel Artis. 2002. "The Regional Distribution of Spanish Unemployment: A Spatial Analysis," Papers in Regional Science, 81, no. 3: 365-389.

Maddala, G. S., and Shaowen Wu. 1999. "A Comparative Study of Unit Root Tests with Panel Data and a New Simple Test," Oxford Bulletin of Economics and Statistics, 61, no. S1: 631-652.

Mayor, Matías, and Ana Jesús López. 2008. "Spatial Shift-Share Analysis Versus Spatial Filtering: An Application to Spanish Employment Data," Empirical Economics, 34, no. 1: 123-142.

Miller, Alan. 2002. Subset Selection in Regression. Boca Raton: Chapman \& Hall/CRC. 
Mitchell, William, and Anthea Bill. 2004. "Spatial Dependence in Regional Unemployment in Australia," in E. Carlson (ed.), A Future that Works: Economics, Employment and the Environment. Proceedings of the 6th Path to Full Employment Conference and the 11th National Conference on Unemployment. Newcastle: The University of Newcastle, pp. 312-326.

Molho, Ian. 1995. "Spatial Autocorrelation in British Unemployment," Journal of Regional Science, 35, no. 4: 641-658.

Moretti, Enrico. 2010. "Local Labor Markets," in TBA (ed.), Handbook of Labor Economics: Elsevier.

Nickell, Stephen. 1981. "Biases in Dynamic Models with Fixed Effects," Econometrica, 49, no. 6: 1417-1426.

Niebuhr, Annekatrin. 2003. "Spatial Interaction and Regional Unemployment in Europe." In European Journal of Spatial Development.

Nijkamp, Peter. 2009. "Regional Development as Self-Organized Converging Growth," in G. Kochendörfer-Lucius and B. Pleskovic (eds.), Spatial Disparities and Development Policy. Washington, DC: World Bank, pp. 265-281.

Oud, Johan H. L., Henk Folmer, Roberto Patuelli, and Peter Nijkamp. 2010. "ContinuousTime Modeling with Spatial Dependence," Geographical Analysis, (forthcoming).

Partridge, Mark D., and Dan S. Rickman. 2010. "Computable General Equilibrium (CGE) Modelling for Regional Economic Development Analysis," Regional Studies, 44, no. 10: 1311-1328.

Patacchini, Eleonora, and Yves Zenou. 2007. "Spatial Dependence in Local Unemployment Rates," Journal of Economic Geography, 7, no. 2: 169-191.

Patuelli, Roberto. 2007. "Regional Labour Markets in Germany: Statistical Analysis of Spatio-Temporal Disparities and Network Structures." Ph.D. Thesis, VU University Amsterdam.

Patuelli, Roberto, Daniel A. Griffith, Michael Tiefelsdorf, and Peter Nijkamp. 2010a. "Spatial Filtering and Eigenvector Stability: Space-Time Models for German Unemployment Data," International Regional Science Review, (forthcoming).

2010b. "Spatial Filtering Methods For Tracing Space-Time Developments In An Open Regional System: Experiments with German Unemployment Data," in A. Frenkel, P. Nijkamp and P. McCann (eds.), Societies in Motion: Regional Development, Industrial Innovation and Spatial Mobility. Cheltenham Northampton: Edward Elgar.

Peng, Roger. 2008. "A Method for Visualizing Multivariate Time Series Data," Journal of Statistical Software, Code Snippets, 25, no. 1: 1-17.

Sarno, Lucio, and Mark P. Taylor. 1998. "Real Exchange Rates under the Recent Float: Unequivocal Evidence of Mean Reversion," Economics Letters, 60, no. 2: 131-137.

Schanne, Norbert, Rüdiger Wapler, and Antje Weyh. 2009. "Regional Unemployment Forecasts with Spatial Interdependencies," International Journal of Forecasting, 26, no. 4: 908-926.

Song, Frank M., and Yangru Wu. 1997. "Hysteresis in Unemployment Evidence from 48 U.S. States," Economic Inquiry, 35, no. 2: 235-243.

SVIMEZ. 2009. "Rapporto SVIMEZ 2009 sull'Economia del Mezzogiorno [SVIMEZ Report 2009 on the Economy of the Mezzogiorno]," SVIMEZ.

Taylor, Jim, and Steve Bradley. 1997. "Unemployment in Europe: A Comparative Analysis of Regional Disparities in Germany, Italy and the UK," Kyklos, 50, no. 2: 221-245.

Tyrowicz, Joanna, and Piotr Wójcik. 2010a. "Nonlinear Stochastic Convergence Analysis of Regional Unemployment Rates in Poland," The Review of Economic Analysis, (forthcoming). 
2010b. "Regional Dynamics of Unemployment in Poland. A Convergence Approach," in F. E. Caroleo and F. Pastore (eds.), The Labour Market Impact of the EU Enlargement: A New Regional Geography of Europe? Berlin Heidelberg: Springer-Verlag, pp. 149-173.

2010c. "Unemployment Convergence in Transition," in E. Marelli and M. Signorelli (eds.), Economic Growth and Structural Features of Transition. London: Palgrave Macmillan.

Zenou, Yves. 2009. Urban Labour Economics. Cambridge: Cambridge University Press. 


\section{Annex}

TABLE 5: Information criteria results

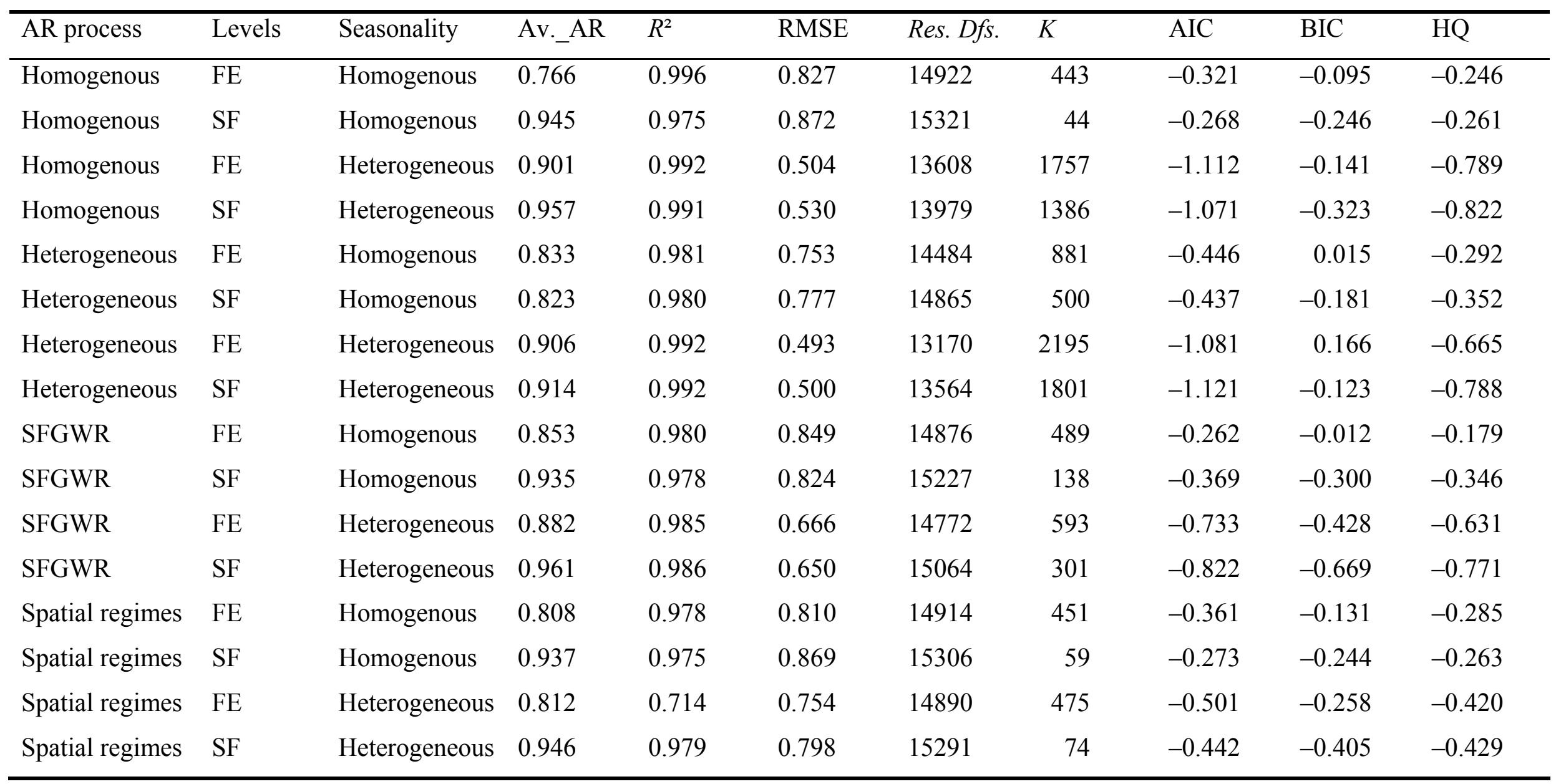




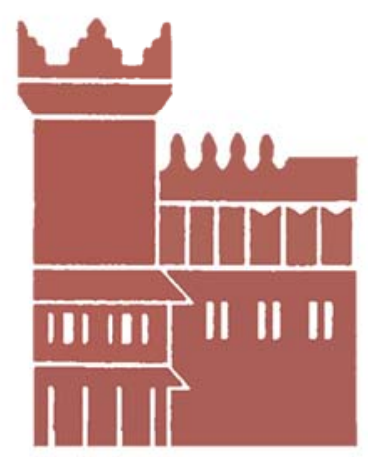

Alma Mater Studiorum - Università di Bologna DEPARTMENT OF ECONOMICS

Strada Maggiore 45

40125 Bologna - Italy

Tel. +39051 2092604

Fax +390512092664

http://www.dse.unibo.it 